\title{
A risk score model with five long non-coding RNAs for predicting prognosis in gastric cancer: an integrated analysis combining TCGA and GEO datasets
}

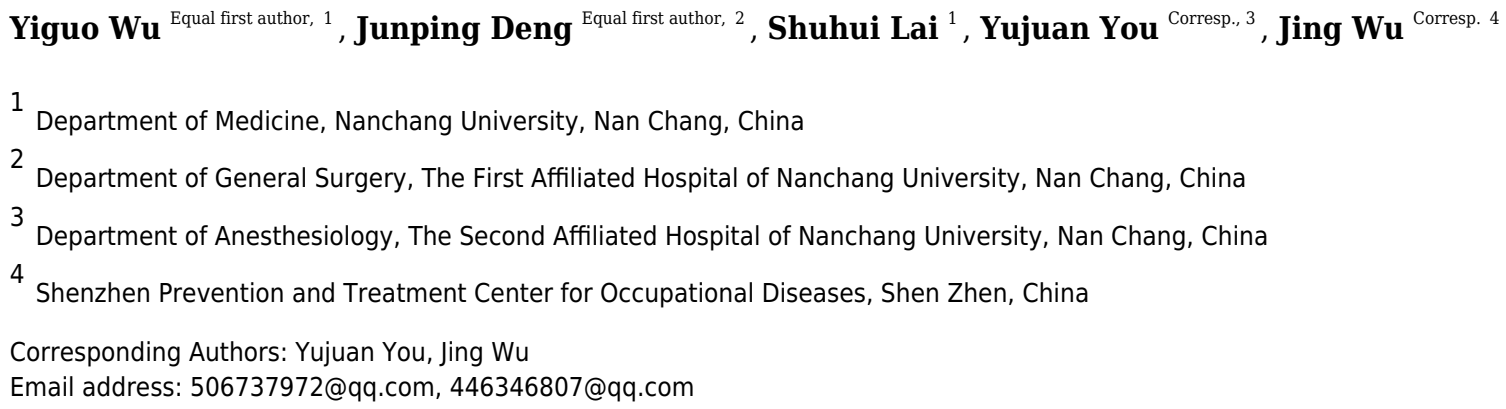

Background. Gastric cancer $(\mathrm{GC})$ is one of the most common carcinomas of the digestive tract, and the prognosis for these patients may be poor. There is evidence that some long non-coding RNAs(IncRNAs) can predict the prognosis of patients with GC. However, few IncRNA signatures have been used to predict prognosis. Herein, we aimed to construct a risk score model based on the expression of five IncRNAs to predict the prognosis of patients with GC and provide new potential therapeutic targets. Methods. We performed differentially expressed and survival analyses to identify differentially expressed survivalralated IncRNAs by using GC patient expression profile data from The Cancer Genome Atlas (TCGA) database. We then established a formula including five IncRNAs to predict the prognosis of patients with GC. In addition, to verify the prognostic value of this risk score model, two independent Gene Expression Omnibus (GEO) datasets, GSE62254 ( $N=300$ ) and GSE 15459 ( $N=200)$ were employed as validation groups. Results. Based on the characteristics of five IncRNAs, patients with GC were divided into high or low risk subgroups. The prognostic value of the risk score model with five IncRNAs was confirmed in both TCGA and the two independent GEO datasets. Furthermore, stratification analysis results showed that this model had an independent prognostic value in patients with stage II-IV GC. We constructed a nomogram model combining clinical factors and the five IncRNAs to increase the accuracy of prognostic prediction. Enrichment analysis based on Kyoto Encyclopedia of Genes and Genomes (KEGG) suggested that the five IncRNAs are associated with multiple cancer occurrence and progression-related pathways.

Conclusion. The risk score model including five IncRNAs can predict the prognosis of patients with GC, especially those with stage II-IV, and may provide potential therapeutic targets in future. 
2 A risk score model with five long non-coding RNAs for 3 predicting prognosis in gastric cancer: an integrated 4 analysis combining TCGA and GEO datasets

5

6

7

8

9

10

Yiguo $\mathrm{Wu}$ Equal first author, 1, Junping Deng Equal first author, 2, Shuhui Lai ${ }^{1}$, Yujuan $\mathrm{You}^{3}$, Jing $\mathrm{Wu}^{4}$

${ }^{1}$ Department of Medicine, Nanchang University, Nanchang, Jiangxi Province, China

${ }^{2}$ Department of General Surgery, The First Affiliated Hospital of Nanchang University, Nanchang, Jiangxi Province, China

${ }^{3}$ Department of Anesthesiology, The Second Affiliated Hospital of Nanchang University, Nanchang, Jiangxi Province, China

${ }^{4}$ Department of Health Surveillance, Shenzhen Prevention and Treatment Center for Occupational Diseases, Shenzhen, Guangdong Province, China

Corresponding Authors:

Yujuan You

No. 1 Minde Road, Nanchang City, Jiangxi Province, 330006, China

Email address: 506737972@qq.com

Jing $\mathrm{Wu}$

No.2019 Buxin Road, Luohu District, Shenzhen City, Guangdong Province, 518020, China

Email address:446346807@qq.com

\section{Abstract}

Background. Gastric cancer (GC) is one of the most common carcinomas of the digestive tract, and the prognosis for these patients may be poor. There is evidence that some long non-coding RNAs(lncRNAs) can predict the prognosis of patients with GC. However, few lncRNA signatures have been used to predict prognosis. Herein, we aimed to construct a risk score model based on the expression of five lncRNAs to predict the prognosis of patients with GC and provide new potential therapeutic targets.

Methods. We performed differentially expressed and survival analyses to identify differentially expressed survival-ralated lncRNAs by using GC patient expression profile data from The Cancer Genome Atlas (TCGA) database. We then established a formula including five lncRNAs to predict the prognosis of patients with GC. In addition, to verify the prognostic value of this risk score model, two independent Gene Expression Omnibus (GEO) datasets, GSE62254 $(N=300)$ and $\operatorname{GSE} 15459(N=200)$ were employed as validation groups.

Results. Based on the characteristics of five lncRNAs, patients with GC were divided into high or low risk subgroups. The prognostic value of the risk score model with five lncRNAs was confirmed in both TCGA and the two independent GEO datasets. Furthermore, stratification analysis results showed that this model had an independent prognostic value in patients with 
41 stage II -IV GC. We constructed a nomogram model combining clinical factors and the five 42 IncRNAs to increase the accuracy of prognostic prediction. Enrichment analysis based on Kyoto 43 Encyclopedia of Genes and Genomes (KEGG) suggested that the five lncRNAs are associated 44 with multiple cancer occurrence and progression-related pathways.

45 Conclusion. The risk score model including five lncRNAs can predict the prognosis of patients 46 with GC, especially those with stage II-IV, and may provide potential therapeutic targets in 47 future.

\section{Introduction}

50

51

52

53

54

55

56

57

58

59

60

61

62

63

64

65

66

67

68

69

70

71

72

73

74

75

76

77

78

79

Gastric cancer (GC) is one of the most common carcinomas of the gastrointestinal (GI) tract and is particularly prevalent in Asian countries. It is estimated that approximately 679,100 individuals were diagnosed with GC in 2015 in China and approximately 498,000 of them died that same year (Saka et al., 2011; Chen et al., 2016). The standard therapies for GC are surgery and chemotherapy. However, most patients with advanced GC show recurrence of the malignancy and metastasis after treatment, resulting in poor prognosis. Despite considerable research in therapies for GC, the prospects of survival of patients with GC remain bleak (Saka et al., 2011). The identification of patients with GC with poor prognosis and the administration of effective treatment as early as possible are key to improving survival. The investigation of potential therapeutic and prognostic biomarkers for GC is of considerable importance.

Long non-coding RNAs (lncRNAs) are RNAs with lengths of $\geq 200$ nucleotides with no or limited protein-coding potential. There is considerable evidence that lncRNAs play crucial roles in the initiation and developments of cancers. For example, lncRNA-ATB disorders contribute to cancer cell proliferation, migration, invasion, and drug-resistance as well as induce epithelialmesenchymal transition by competitively binding to microRNAs(Li et al., 2017; Balas \& Johnson, 2018). Some researchers have suggested that lncRNAs serve as new prognostic biomarkers in various cancers, including CCAT2 (Yu et al., 2017), HOXB-AS3 (Huang et al., 2017), and ASLNC07322 (Li et al., 2019) in colon cancer. Many lncRNAs closely related to the prognosis of patients with GC have been identified, including MEG3(Wei \& Wang, 2017), SNHG7(Wang et al., 2017), and DANCR(Mao et al., 2017)(Mao et al., 2017) ${ }^{[10][10][10](M a o ~ e t ~ a l ., ~}$ 2017). Risk score models have also been constructed to predict the prognosis of human cancers. The differences in prognosis in non-small-cell lung cancer can be identified by its eight-lncRNA signature (Miao et al., 2019). However, the identification of lncRNAs related to the prognosis of patients with GC is still in its early stages and additional research is warranted.

In this study, we analyzed the data of 450 patients with GC from The Cancer Genome Atlas (TCGA) database to identify differentially expressed lncRNAs for the prognostic prediction. We used two independent Gene Expression Omnibus (GEO) (Barrett et al., 2013) datasets to validate the selected-lncRNAs. In addition, we analyzed the accuracy of the prediction of five lncRNAs in different clinical subgroups using lncRNA data in combination with the clinical characteristics of the patients. Furthermore, we constructed a nomogram model by combining clinical factors 
80

81

82

83

84

85

86

87

88

89

90

91

92

93

94

95

96

97

98

99

100

101

102

103

104

105

106

107

108

109

110

111

112

113

114

115

116

117

118

119

and five lncRNAs to increase the accuracy of prognostic prediction. Finally, we performed pathway enrichment analysis to determine the potential functions of these lncRNAs in GC.

\section{Materials \& Methods}

\section{Preparation of GC datasets}

We acquired a training dataset of GC samples from TCGA comprising 450 samples and 14147 lncRNAs (case: normal $=414: 36$ ). We used these 450 samples to perform differential expression analysis. After excluding six cases with missing overall survival (OS) prognostic information, 408 cases were included for further univariate Cox proportional hazard regression analysis and subsequent analysis in the training group. The microarray data for the validation group and survival data of the patients are publicly available at GEO with accession numbers GSE62254 ( $N$ $=300 ; 1397$ lncRNAs $)$ and GSE15459 $(N=200 ; 1397$ lncRNAs $)$.

\section{Normalization of GEO data}

Because the two GEO datasets (GSE62254 and GSE15459) had different expression profiles, we performed quantile normalization on the original data and downloaded it as a probe-level CEL file. Affymetrix U133 Plus 2.0 was used as the probe matching platform. Data were downloaded from the Affymetrix website (http://www.affymetrix.com), and a total of 2986 IncRNA-specific probes were included.

\section{Construction of an IncRNA-based risk score model from the training group}

The IncRNAs that were differentially expressed between GC and normal gastric tissue in TCGA dataset were identified using the "limma" $\mathrm{R}$ package of the $\mathrm{R}$ statistical computing environment $(\log 2 \mid$ fold change $\mid>1$ and adjusted $P<0.05)$, and the adjusted $P$ value was used to reduce false positives(Deng et al., 2019; Zeng et al., 2019). The candidate lncRNAs were analyzed using univariate Cox proportional hazard regression analysis $(P<0.05)$. The cutoff values of lncRNA expression were determined as the median of all expression values in Cox survival analysis. In total, we identified $278 \mathrm{lncRNAs}$ with statistically significant differences. After identifying the lncRNAs common to both TCGA and GEO (GSE62254) datasets, we performed multivariate Cox proportional hazards analysis to identify independent prognostic lncRNAs. Finally, we constructed an lncRNA-based risk score model from a linear combination of the expression levels of these lncRNAs, multiplied by the regression coefficients obtained from the multivariate Cox proportional hazards regression analysis.

\section{Validation of the IncRNA-based model for prognostic prediction}

We calculated the risk scores of each patient and used the corresponding median score as the cutoff value to classify them into two groups: high risk and low risk subgroups. We used KaplanMeier analysis to compare the survival of the two groups and time-dependent receiver operating characteristic (ROC) curves to assess our lncRNA-based risk model. We used two GEO datasets to validate the model for prognostic prediction. Cox proportional hazards regression analysis was used to estimate the hazard ratio (HR) of the model with $95 \%$ confidence intervals to further evaluate the predictive value of the model for each clinical subgroup. Clinical subgroups were determined based on sex, tumor-node-metastases (TNM) stage, histologic grade, race, and age. 
120 Finally, we constructed a nomogram combining the model with clinical factors using the "rms"

121

122

123

124

125

126

127

128

129

130

131

132

133

134

135

136

137

138

139

140

141

142

143

144

145

146

147

148

149

150

151

152

153

154

155

156

157

158

package. We also calculated the concordance index (C-index) and plotted a calibration curve to determine its predictive accuracy and discriminatory capacity.

\section{Potential functions of the five IncRNAs}

To determine the potential functions of the five lncRNAs, which appeared to be discriminatory, we performed linear regression analysis of the relationship between the lncRNAs and the protein-coding genes in TCGA dataset. The screening criteria for the protein-coding genes were a positive association with at least one lncRNA (Pearson coefficient $>0.4$ ). After identifying the candidate genes, we screened out aberrantly activated signaling pathways using the Kyoto Encyclopedia of Genes and Genomes (KEGG) enrichment analysis via web-based Gene Set Analysis Toolkit (http://www.webgestalt.org/), a popular software tool for functional enrichment analysis related to KEGG pathways(Yang et al., 2019; Wang et al., 2013),.

\section{Statistical analysis}

We used the R software (version 3.6.1) for statistical analyses. Differentially expressed analysis was performed using the "limma" $\mathrm{R}$ package. Univariate and multivariate Cox proportional hazards regression analyses were performed to identify prognosis-related lncRNAs. The "survival" and "survminer" packages were used for Cox proportional hazards regression analyses, Kaplan-Meier survival analysis and calculation of C-index. A time-dependent ROC curve to assess the specificity and sensitivity of the risk score model was constructed using the "survivalROC" package. A nomogram combining the risk score model with the clinical factors was constructed using the "rms" package. The Review Manager software (version 5.3) was used to construct a forest plot. Chi-square tests were used to compare the recurrence and mortality rates between the high and low risk subgroups. A $P$-value of $<0.05$ was considered statistically significant, and all tests were two sided. Pearson's linear regression analysis was used to determine the relationship between lncRNAs and protein-coding genes.

\section{Results}

\section{Identification of five prognostic IncRNAs}

The datasets are publicly available and recruitment has already happened. We performed differentially expressed analysis $(\log 2 \mid$ fold change $\mid>1$ and adjusted $P<0.05)$ and univariate Cox proportional hazard regression analysis $(P<0.05)$ to identify survival-related lncRNAs. A total of 278 lncRNAs were analyzed further. To validate the predictive accuracy, we compared the IncRNAs selected from TCGA database with the GEO validation group. We found that 37 IncRNAs were common between the 278 lncRNAs and the validation dataset (GSE62254) (Table S1). Multivariate Cox proportional hazards regression analyses identified five lncRNAs as independent prognostic factors of GC: LINC00205, TRHDE-AS1, OVAAL, LINC00106, and MIR100HG (Table 1). Fig. 1A-B shows the expression profiles of the five lncRNAs in patients with GC as volcano and heat maps, and Fig. 1C-D shows the survival curves based on the OS and disease-free survival (DFS) of the 408 patients. Owing to the lack of clinical data in 
159 GSE15459, Table 2 shows the clinical features of patients with GC in the training group and 160 GSE62254.

161 Construction of an IncRNA-based risk model from the training group

162

163

164

165

166

167

168

169

170

171

172

173

174

175

176

177

178

179

180

181

182

183

184

185

186

187

188

189

190

191

192

193

194

195

196

197

198

According to the schematic workflow of this study (Table 3), using the coefficients of the five lncRNAs identified by multivariate Cox hazard analysis, we created a risk-score formula as follows: risk score $=(0.249092 \times$ expression level of LINC00205 $)+(0.182045 \times$ expression level of TRHDE-AS1 $)+(0.271169 \times$ expression level of OVAAL $)+(-0.20794 \times$ expression level of LINC00106) $+(0.502539 \times$ expression level of MIR100HG). Among the five lncRNAs, LINC00106, which had a negative coefficient, was considered as a protective factor. The remaining four IncRNAs with positive coefficients, namely LINC00205, TRHDE-AS1, OVAAL, and MIR100HG, were risk factors. The risk scores of each patient in the training group were calculated (Table S2), ranging from -2.086959745 to 2.270305234 . The patients in the training group were divided into two subgroups: high risk $(n=204)$ and low risk $(n=204)$ subgroups, with the median score $(-0.001085)$ as the cutoff value. We performed Kaplan-Meier survival analysis to assess the effect of the lncRNA-based risk model on the OS and DFS of patients with $\mathrm{GC}$ in the training group (Fig. 2A-B). Our results indicated that the high-risk group had a significantly poorer prognosis than the low risk group for both OS and DFS $\left(P=1 \times 10^{-6}\right.$ and $6 \times$ $10^{-6}$, respectively). Fig. $2 \mathrm{C}-\mathrm{F}$ shows the scatter plots of the recurrence and mortality rates of patients with GC. The recurrence and mortality rates were significantly higher in the high risk group than in the low risk group $(P<0.001)$. To accurately evaluate the prognostic value of the five-lncRNA signature, we performed time-dependent ROC analysis using the 1-4 years cutoff value of OS and the 1-2 years cutoff of DFS as the ROC ending points (Fig. 2G-H and Fig. S1AD). The area under the ROC curve (AUC) was 0.734 for the 4-year cutoff value of OS and 0.692 for the 2-year cutoff value of DFS, respectively, and had the highest predictive value among those years, indicating that our model can be used for survival prediction in patients with GC (Fig. 2G-H).

\section{Validation of the IncRNA-based risk score model for prognostic prediction in independent groups}

To assess the prognostic significance of this novel lncRNA-based risk model including the five-IncRNA signature in patients with GC, we used the other two independent validation datasets from the GEO database. We calculated the risk score using the formula mentioned above (Table S2). The patients with GC in GSE62254 (validation group-1; $N=300$ ) and GSE15459 (validation group-2; $N=200$ ) datasets were divided into high risk and low risk groups according to the median risk score. Owing to the lack of DFS data in GSE15459, we only calculated the OS of the patients. The high risk group had a poorer OS than the low risk group (log-rank $P=0.01)$ (Fig. 3A-B). Figure 3C-D shows the scatter plots for the death events. The mortality rates were significantly higher in the high risk group than in the low risk group $(P<$ 0.001 ). The AUC for the two validation groups in the 4-year cutoff OS was 0.622 and 0.610 for validation group 1 and 2, respectively (Fig. 3E-F). Figure S2A-F shows the ROC curve for the 1-3 year cutoff OS for the validation groups 1 and 2. Furthermore, we verified the performance

Peer) reviewing PDF | (2020:02:45656:3:0:NEW 4 Nov 2020) 
199

200

201

202

203

204

205

206

207

208

209

210

211

212

213

214

215

216

217

218

219

220

221

222

223

224

225

226

227

228

229

230

231

232

233

234

235

236

237

of our risk score model for DFS of the GSE62254 dataset (Fig. S3A-D). Our results further confirmed the value and robustness of this risk score model for prognostic prediction in patients with GC.

\section{The IncRNA-based risk model has a favorable prognostic prediction in patients with stage} II, III, and IV

To further investigate the performance of our lncRNA-based risk model, we performed stratified Kaplan-Meier survival analysis of OS in the training group based on the AJCC TNM stages I, II, III, or IV (Fig. 4A-D). The five-lncRNA signature showed good predictive value for OS of stages II $(P=0.008)$, III $(P=0.02)$, and IV $(P=0.01)$, but not I $(P=0.3)$.

To estimate the HR of each subgroup of patients as defined by sex, TNM stage, histologic grade, race and age $(\geq$ or $<50$ years) (Table 4$)$, we used our model to divide the patients into two risk groups on the basis of the median cutoff value. Forest plots are shown in Figure 5. Table S3 shows the HR of each subgroup of patients in GSE62252. The risk score model had a relatively good prognostic value in the clinical subgroups of sex, histologic grade and age. To improve the prognostic value of this model, we combined the clinical factors with the risk score model to construct a nomogram model for prognostic prediction. The nomogram model and calibration curve are shown in Figure 6A-B. To evaluate the effect of the nomogram model, we calculated its C-index. The C-index for predicting the 4-year OS of patients with GC was 0.69668, indicating that this model is a valuable indicator for prognostic prediction.

\section{Potential functions of the five IncRNAs}

To investigate the functions of the five lncRNAs in patients with GC, we calculated Pearson correlations between the five-lncRNA signature and 19605 protein-coding genes in TCGA dataset. A total of 3069 genes (Table S4) were positively correlated with at least one lncRNA (Pearson's coefficient> 0.4) (Fig. 7A). We further selected these genes for KEGG pathway enrichment analysis. By ranking based on $-\log P$ value $(Q$ value), we selected the top 10 pathways for construction of a bubble plot (Fig. 7B) (Zeng et al., 2019; Deng et al., 2019). For biological processes, the co-expressed genes were mainly enriched in pathways involved in cancer, such as the focal adhesion pathway, cGMP-PKG signaling pathway and calcium signaling pathway. This finding indicates that the five lncRNAs may be involved in the regulation of tumor initiation and progression.

\section{Discussion}

In this study, we identified a potential signature involving five lncRNAs that are differentially expressed in tumor and normal tissues, and might be valuable for prognostic prediction in GC. The prognostic performance of this lncRNA-based risk score model was verified using both TCGA and GEO datasets. Stratified analysis suggested that the risk score model is valuable for prognostic prediction in patients with stage II-IV GC. To enhance the predictive accuracy of the model, we combined clinical parameters with the five-lncRNA signature to construct a nomogram model and confirmed its performance using a calibration curve and $\mathrm{C}$ index. 
238

239

240

241

242

243

244

245

246

247

248

249

250

251

252

253

254

255

256

257

258

259

260

261

262

263

264

265

266

267

268

269

270

271

272

273

274

275

276

277

GC is a common malignancy of the GI tract (Siegel et al., 2019). Despite continuous improvements in treatment, the 5-year survival rate of patients with advanced GC is only approximately 20\% (Min et al., 2019; Misawa et al., 2019). Therefore, early diagnosis, early identification of high-risk patients and implementation of effective treatment measures as early as possible are necessary to improve survival. It is also important to develop novel prognostic indicators of GC. Over the past few decades, research has shown that protein-coding genes(Ghoorun et al., 2019; Luo et al., 2019) and microRNAs(Li et al., 2020; Zhou et al., 2019), play vital roles in the occurrence and development of various cancers, and can also be used to predict patient prognosis. Several nomogram models involving clinical factors have been constructed to predict the prognosis of patients with GC. For example, Yu (Yu \& Zhang, 2019)used tumor size and tumor site, as independent prognostic factors, to construct OS nomograms for predicting outcomes in patients with $\mathrm{GC}$, and the $\mathrm{C}$-index of this model indicated that it could predict prognosis. Recently, more lncRNAs related to GC prognosis have been discovered; however, prognostic prediction models involving lncRNAs still lack consensus. We present a nomogram including clinical factors and the five-lncRNA signature that might be of value for prognostic prediction in GC.

It is necessary to explore novel biomarkers to improve the diagnostic accuracy and prognosis of GC because of limitations of TNM staging and some related scoring systems. Many lncRNAs have been identified, of which only few have been functionally annotated. However, evidence indicates that lncRNAs, acting either as oncogenes or tumor suppressors, participate in the tumorigenesis and development of various cancers by regulating chromatin remodeling, transcription and post-transcriptional modification(Bartonicek et al., 2016; Iyer et al., 2015), and therefore might be valuable for cancer diagnosis and prognosis. Some studies have found that GC-related lncRNAs are involved in biological behaviors including the proliferation, migration, invasion, and autophagy of GC cells, thereby affecting the initiation and prognosis of GC (Mao et al., 2017; Wei \& Wang, 2017). For example, the lncRNA MEG3 inhibits the proliferation, metastasis, and prognosis of GC cells by upregulating the expression p53-a key tumor suppressor (Wei \& Wang, 2017). We identified five lncRNAs-LINC00205, TRHDE-AS1, OVAAL, LINC00106, and MIR100HG - as predictors of GC prognosis, and developed a riskscore model. Kaplan-Meier analysis suggested that our lncRNA-based risk model is valuable for predicting GC prognosis. We used two independent GEO datasets as validation datasets. Our results confirmed that our risk score model is stable and performs well in the prognostic prediction of GC.

Of the five IncRNAs, LINC00205, TRHDE-AS1, OVAAL, and MIR100HG, act as risk factors of GC, whereas LINC00106 is a protective factor. Apart from LINC00205 and MIR100HG, the other three lncRNAs have not been reported much in the literature. Our study identified LINC00205, TRHDE-AS1, OVAAL, and MIR100HG as potential prognostic biomarkers of $\mathrm{GC}$ for the first time. Consistent with our result, it has previously been reported that high expression of LINC00106 indicates prolonged OS of patients with GC (Qi et al., 2020). Nevertheless, the role of this lncRNA in GC as well as its specific mechanism need to be further

Peer) reviewing PDF | (2020:02:45656:3:0:NEW 4 Nov 2020) 
278 investigated. Interestingly, in hepatocellular carcinoma (HCC), comprehensive genome-wide 279 analysis revealed that the expression of LINC00205, a tumor suppressor, is positively associated 280 with OS and recurrence-free survival (Cui et al., 2017). A study showed that, as a competing 281 endogenous RNA with lower expression levels in tumor tissues, LINC00205 may negatively 282 regulate HCC progression via the miR-184/EPHX1 axis (Long et al., 2019), While another study 283 indicated that LINC00205, can serve as an oncogene, and can promote the proliferation,

284

285

286

287

288

289

290

291

292

293

294

295

296

297

298

299

300

301

302

303

304

305

306

307

308

309

310

311

312

313

314

315

316

317 migration and invasion of HCC cells by targeting miR-122-5p(Zhang et al., 2019). In addition, LINC00205 can act as a protective factor in pancreatic cancer $[\mathrm{HR}=0.58, P(\log$ rank $)=$ 0.0091] (Giulietti et al., 2018). The reported role and therefore prognostic prediction value of LINC00205 in various cancers shows significant discrepancies. These discrepancies might be associated with the specificities of different cancers. The upregulation of TRHDE-AS1 inhibits the growth of lung carcinoma through competitive combination with the miRNA-103-KLF4 axis (Zhuan et al., 2019). A study has found that OVVAL is highly expressed in colon cancer and melanoma, and further experimental results showed that OVAAL promotes the proliferation of cancer cells via dual mechanisms controlling RAF/MEK/ERK signaling and p27-mediated cell senescence (Sang et al., 2018). The lncRNA MIR100HG has been studied as an oncogene in acute megakaryoblastic leukemia (Emmrich et al., 2014), and laryngeal squamous cell carcinoma (Huang et al., 2019), as well as for its role in mediating cetuximab resistance via Wnt/ $\beta$-catenin signaling (Lu et al., 2017) in colorectal cancer. Although the roles of these lncRNAs in cancer need to be further investigated, our results may provide a novel approach to study GC.

To further investigate the functions of the five lncRNAs in GC, we performed pathway enrichment analysis. These genes are enriched in cancer regulation, including the cGMP-PKG signaling pathway, calcium signaling pathway, and focal adhesion pathway etc. This finding suggests that the five lncRNAs may play an important role in the occurrence and development of GC. There is evidence that IncRNAs can promote tumorigenesis through the cGMP-PKG signaling pathway. For example, the overexpression of SRRM2-AS accelerates angiogenesis in nasopharyngeal carcinoma via the cGMP-PKG signaling pathway (Chen et al., 2019). The calcium signaling pathway has been reported to be mainly involved in metabolic diseases and heart diseases over the past years (Berridge, 2016; Dewenter et al., 2017). A recent research showed that the calcium signaling pathway was associated with cancer cell survival, but more details on its effects remain to be studied (Reczek \& Chandel, 2018). Focal adhesion sites are special sites where integrin receptors aggregated in cells interact with extracellular matrix and intracellular actin skeleton (Burridge, 2017), and they play a critical role in tumor invasion and migration (Shen et al., 2018). There is evidence that knockdown of Linc01060 could promote the progression of pancreatic cancer via the vinculin-mediated focal adhesion pathway turnover (Shi et al., 2018). However, whether the lncRNA can mediate the progression of GC through the focal adhesion pathway is less reported. In short, lncRNAs may participate in the genesis and development of various tumors via the above pathways.

Risk score model is a common and widely used method to predict the prognosis of patients with multiple diseases (Lemke et al., 2017; Li et al., 2018; Yang et al., 2017; Sobotka et al., 
318 2018). Our risk score was determined by the expression of independent survival-lncRNAs 319 obtained after Cox hazard analyses and its corresponding coefficients. It was calculated using

320

321

322

323

324

325

326

327

328

329

330

331

332

333

334

335

336

337

338

339

340

341

342

343

344

345

346

347

348

349

350

351

352

353

354

355

356

357 binary lncRNA expression values according to the medians of original lncRNA expression values. This adjustment helps to improve the clinical application of the prognostic model in other study population (Zhang et al., 2018). In general, the higher is the risk score, the poorer is the prognosis, which is consistent with our analysis. Our Kaplan-Meier survival analysis showed that the patients in the high risk group had a significantly poorer prognosis than those in the low risk group. Our risk score model based on lncRNAs has several advantages. This model based on the expression of five lncRNAs provides a novel noninvasive method for predicting the prognosis of patients with GC before surgery. Compared with conventional invasive pathological examinations, it reduces unnecessary pain for patients. Second, this five-lncRNA risk model can provide preoperative risk predictive probability of individual mortality and recurrence in different clinical endpoints. It is simple and convenient for clinicians and patients to understand. Third, our model used the median of five-lncRNA-based risk score as the cutoff value to divide patients into high risk and low risk groups. It can identify patients at high risk of mortality or recurrence in a timely manner and prompt clinical interventions as early as possible to improve their prognosis.

There have been several reports on lncRNA signatures for GC. A previous study reported a 24-lncRNA signature that can predict outcomes in patients with GC by applying the random survival forest-variable hunting algorithm using GEO datasets (Zhu et al., 2016). However, because of the limited amount of data in the GEO datasets, the lncRNAs identified in this study might not represent the complete population of lncRNAs involved in GC. In this study, we integrated 950 samples from TCGA and GEO databases to comprehensively investigate the potentially prognostic lncRNAs. This greatly improved the accuracy, reliability and robustness of our model. A six-lncRNA prognostic signature was established by robust likelihood-based survival and LASSO model (Ma et al., 2019). Whether the six-lncRNA signature combined with other clinical features can enhance the predictive power remains to be determined. To improve the accuracy of the five-lncRNA prognosis model, we combined it with clinical factors to develop a nomogram model that could predict the OS of patients with GC. Zhu (Zhu et al., 2018) et al constructed an 11-IncRNA signature by univariate and multivariate Cox regression analyses. Although an internal validation was validated using the bootstrap resampling method, external validation studies are needed to further evaluate the value of this model. We not only included two external verification datasets, but also performed survival analysis, ROC curve analysis, and constructed a forest plot for predictive verification, indicating a favorable effectiveness of our model.

There are some limitations of the present study. We integrated data from TCGA and GEO databases to increase the number of the cases, thereby reducing bias from a small sample size. Integrated analysis has been proved to be an effective approach for multiple datasets with different platforms using R package (Zhang et al., 2019; Nie et al., 2020; Zhao et al., 2018; Zhao et al., 2020), thereby promoting the reliability of our conclusion (Ma et al., 2017). However, 
358

359

360

361

362

363

364

365

366

367

368

369

370

371

372

373

374

375

376

377

378

379

380

381

382

383

384

385

386

387

388

389

390

391

392

393

394

395

396

397

398

399

400

401

402

403

TCGA dataset has a larger number of lncRNAs than the GEO dataset (14147:1397) because of different sequencing technologies: TCGA uses RNA sequencing technology, whereas GEO uses microarray chip technology. Intersection of three datasets has inevitably omitted potential prognostic IncRNAs. Moreover, the clinical characteristics of the patients in the three datasets are heterogeneous. This might have inevitably led to a bias. Besides, owing to the lack of DFS and clinical data in GSE15459, we used only one external validation group to verify the prognostic value of the five-lncRNA signature for the DFS of patients. In addition, many important variables affecting the prognosis of patients with GC are not provided in TCGA and GEO datasets, such as dietary habits, previous disease, history of chemotherapy or radiation therapy, and family history of cancer. Thus, on the one hand, it is necessary to perform a largescale multi-center prospective clinical study based on the same sequencing technology to decrease the bias mentioned above. On the other hand, based on existing data, it is beneficial to develop innovative statistical algorithms to reduce the heterogeneity of different data sources. Last, because of the limited number of studies regarding these lncRNAs, experimental research on these lncRNAs is highly warranted to further understand their functions in GC.

\section{Conclusions}

We established a risk score model including five lncRNAs to predict the OS and DFS of patients with GC, particularly in those with stage II -IV GC. Our findings also provided evidence of developing effective prognostic biomarkers for patients with GC and potential therapeutic targets in the future.

\section{Acknowledgements}

At the point of finishing this paper, we would thank to Jie Peng for technical assistance with the data analysis.

\section{References}

Balas, M.M., and Johnson, A.M. 2018. Exploring the mechanisms behind long noncoding RNAs and cancer. Noncoding RNA Res 3:108-117. 10.1016/j.ncrna.2018.03.001

Barrett, T., Wilhite, S.E., Ledoux, P., Evangelista, C., Kim, I.F., Tomashevsky, M., Marshall, K.A., Phillippy, K.H., Sherman, P.M., Holko, M., Yefanov, A., Lee, H., Zhang, N., Robertson, C.L., Serova, N., Davis, S., and Soboleva, A. 2013. NCBI GEO: archive for functional genomics data sets--update. NUCLEIC ACIDS RESEARCH 41:D991D995. 10.1093/nar/gks1193

Bartonicek, N., Maag, J.L., and Dinger, M.E. 2016. Long noncoding RNAs in cancer: mechanisms of action and technological advancements. Molecular Cancer 15:43. 10.1186/s12943-016-0530-6

Berridge, M.J. 2016. The Inositol Trisphosphate/Calcium Signaling Pathway in Health and Disease. PHYSIOLOGICAL REVIEWS 96:1261-1296. 10.1152/physrev.00006.2016

Burridge, K. 2017. Focal adhesions: a personal perspective on a half century of progress. FEBS Journal 284:33553361. 10.1111/febs. 14195

Chen, S., Lv, L., Zhan, Z., Wang, X., You, Z., Luo, X., and You, H. 2019. Silencing of long noncoding RNA SRRM2-AS exerts suppressive effects on angiogenesis in nasopharyngeal carcinoma via activating MYLKmediated cGMP-PKG signaling pathway. JOURNAL OF CELLULAR PHYSIOLOGY. 10.1002/jcp.29382

Chen, W., Zheng, R., Baade, P.D., Zhang, S., Zeng, H., Bray, F., Jemal, A., Yu, X.Q., and He, J. 2016. Cancer statistics in China, 2015. CA: A Cancer Journal for Clinicians 66:115-132. 10.3322/caac.21338

Cui, H., Zhang, Y., Zhang, Q., Chen, W., Zhao, H., and Liang, J. 2017. A comprehensive genome-wide analysis of long noncoding RNA expression profile in hepatocellular carcinoma. Cancer Med 6:2932-2941. 10.1002/cam4.1180

Deng, J., Xu, Y., and Wang, G. 2019. Identification of Potential Crucial Genes and Key Pathways in Breast Cancer Using Bioinformatic Analysis. Frontiers in Genetics 10. 10.3389/fgene.2019.00695 
404

405

406

407

408

409

410

411

412

413

414

415

416

417

418

419

420

421

422

423

424

425

426

427

428

429

430

431

432

433

434

435

436

437

438

439

440

441

442

443

444

445

446

447

448

449

450

451

452

453

454

455

456

457

458

Dewenter, M., von der Lieth, A., Katus, H.A., and Backs, J. 2017. Calcium Signaling and Transcriptional Regulation in Cardiomyocytes. CIRCULATION RESEARCH 121:1000-1020. 10.1161/CIRCRESAHA.117.310355 Emmrich, S., Streltsov, A., Schmidt, F., Thangapandi, V.R., Reinhardt, D., and Klusmann, J.H. 2014. LincRNAs MONC and MIR100HG act as oncogenes in acute megakaryoblastic leukemia. Molecular Cancer 13:171. 10.1186/1476-4598-13-171

Ghoorun, R.A., Wu, X.H., Chen, H.L., Ren, D.L., and Wu, X.B. 2019. Prognostic Significance of FKBP14 in Gastric Cancer. Onco Targets Ther 12:11567-11577. 10.2147/OTT.S221943

Giulietti, M., Righetti, A., Principato, G., and Piva, F. 2018. LncRNA co-expression network analysis reveals novel biomarkers for pancreatic cancer. CARCINOGENESIS 39:1016-1025. 10.1093/carcin/bgy069

Huang, J.Z., Chen, M., Chen, Gao, X.C., Zhu, S., Huang, H., Hu, M., Zhu, H., and Yan, G.R. 2017. A Peptide Encoded by a Putative lncRNA HOXB-AS3 Suppresses Colon Cancer Growth. MOLECULAR CELL 68:171-184. 10.1016/j.molcel.2017.09.015

Huang, Y., Zhang, C., and Zhou, Y. 2019. LncRNA MIR100HG promotes cancer cell proliferation, migration and invasion in laryngeal squamous cell carcinoma through the downregulation of miR-204-5p. Onco Targets Ther 12:2967-2973. 10.2147/OTT.S202528

Iyer, M.K., Niknafs, Y.S., Malik, R., Singhal, U., Sahu, A., Hosono, Y., Barrette, T.R., Prensner, J.R., Evans, J.R., Zhao, S., Poliakov, A., Cao, X., Dhanasekaran, S.M., Wu, Y.M., Robinson, D.R., Beer, D.G., Feng, F.Y., Iyer, H.K., and Chinnaiyan, A.M. 2015. The landscape of long noncoding RNAs in the human transcriptome. NATURE GENETICS 47:199-208. 10.1038/ng.3192

Lemke, M., Law, C.H., Li, J., Dixon, E., Tun, A.M., Hernandez, A.R., Bennett, S., Martel, G., and Karanicolas, P.J. 2017. Three-point transfusion risk score in hepatectomy. Br J Surg 104:434-442. 10.1002/bjs.10416

Li, C.I., Li, T.C., Liu, C.S., Liao, L.N., Lin, W.Y., Lin, C.H., Yang, S.Y., Chiang, J.H., and Lin, C.C. 2018. Risk score prediction model for dementia in patients with type 2 diabetes. EUROPEAN JOURNAL OF NEUROLOGY 25:976-983. 10.1111/ene.13642

Li, J., Li, Z., Zheng, W., Li, X., Wang, Z., Cui, Y., and Jiang, X. 2017. LncRNA-ATB: An indispensable cancerrelated long noncoding RNA. Cell Prolif 50. 10.1111/cpr.12381

Li, J.P., Zhang, H.M., Liu, M.J., Xiang, Y., Li, H., Huang, F., Li, H.H., Dai, Z.T., Gu, C.J., Liao, X.H., and Zhang, T.C. 2020. miR-133a-3p/FOXP3 axis regulates cell proliferation and autophagy in gastric cancer. JOURNAL OF CELLULAR BIOCHEMISTRY. 10.1002/jcb.29613

Li, X., Lv, X., Li, Z., Li, C., Li, X., Xiao, J., Liu, B., Yang, H., and Zhang, Y. 2019. Long Noncoding RNA ASLNC07322 Functions in VEGF-C Expression Regulated by Smad4 during Colon Cancer Metastasis. Mol Ther Nucleic Acids 18:851-862. 10.1016/j.omtn.2019.10.012

Long, X., Li, Q., Zhi, L.J., Li, J.M., and Wang, Z.Y. 2019. LINC00205 modulates the expression of EPHX1 through the inhibition of miR - 184 in hepatocellular carcinoma as a ceRNA. JOURNAL OF CELLULAR PHYSIOLOGY 235:3013-3021. 10.1002/jcp.29206

Lu, Y., Zhao, X., Liu, Q., Li, C., Graves-Deal, R., Cao, Z., Singh, B., Franklin, J.L., Wang, J., Hu, H., Wei, T., Yang, M., Yeatman, T.J., Lee, E., Saito-Diaz, K., Hinger, S., Patton, J.G., Chung, C.H., Emmrich, S., Klusmann, J.H., Fan, D., and Coffey, R.J. 2017. IncRNA MIR100HG-derived miR-100 and miR-125b mediate cetuximab resistance via Wnt/beta-catenin signaling. NATURE MEDICINE 23:1331-1341. 10.1038/nm.4424

Luo, X., He, Y., Tang, H., Cao, Y., Gao, M., Liu, B., and Hu, Z. 2019. Effects of HER2 on the invasion and migration of gastric cancer. American Journal of Translational Research 11:7604-7613.

Ma, B., Li, Y., and Ren, Y. 2019. Identification of a 6 - IncRNA prognostic signature based on microarray re - annotation in gastric cancer. Cancer Medicine 9:335-349. 10.1002/cam4.2621

Ma, T., Liang, F., Oesterreich, S., and Tseng, G.C. 2017. A Joint Bayesian Model for Integrating Microarray and RNA Sequencing Transcriptomic Data. JOURNAL OF COMPUTATIONAL BIOLOGY 24:647-662. $10.1089 / \mathrm{cmb} .2017 .0056$

Mao, Z., Li, H., Du B, Cui, K., Xing, Y., Zhao, X., and Zai, S. 2017. LncRNA DANCR promotes migration and invasion through suppression of lncRNA-LET in gastric cancer cells. Biosci Rep 37. 10.1042/BSR20171070

Miao, R., Ge, C., Zhang, X., He, Y., Ma, X., Xiang, X., Gu, J., Fu, Y., Qu, K., Liu, C., Wu, Q., and Lin, T. 2019. Combined eight-long noncoding RNA signature: a new risk score predicting prognosis in elderly non-small cell lung cancer patients. Aging (Albany NY) 11:467-479. 10.18632/aging.101752

Min, S.H., Won, Y., Kim, G., Lee, Y., Park, Y.S., Ahn, S.H., Park, D.J., and Kim, H.H. 2019. 15 -year experience of laparoscopic gastrectomy in advanced gastric cancer: analysis on short-term and long-term oncologic outcome. SURGICAL ENDOSCOPY AND OTHER INTERVENTIONAL TECHNIQUES. 10.1007/s00464-019-07292-x

Misawa, K., Mochizuki, Y., Sakai, M., Teramoto, H., Morimoto, D., Nakayama, H., Tanaka, N., Matsui, T., Ito, Y.,

Peer) reviewing PDF | (2020:02:45656:3:0:NEW 4 Nov 2020) 
459

460

461

462

463

464

465

466

467

468

469

470

471

472

473

474

475

476

477

478

479

480

481

482

483

484

485

486

487

488

489

490

491

492

493

494

495

496

497

498

499

500

501

502

503

504

505

506

507

508

509

510

511

512

513

514

Ito, S., Tanaka, K., Uemura, K., Morita, S., and Kodera, Y. 2019. Randomized clinical trial of extensive intraoperative peritoneal lavage versus standard treatment for resectable advanced gastric cancer (CCOG 1102 trial). Br J Surg 106:1602-1610. 10.1002/bjs.11303

Nie, K., Deng, Z., Zheng, Z., Wen, Y., Pan, J., Jiang, X., Yan, Y., Liu, P., Liu, F., and Li, P. 2020. Identification of a 14-IncRNA Signature and Construction of a Prognostic Nomogram Predicting Overall Survival of Gastric Cancer. DNA AND CELL BIOLOGY. 10.1089/dna.2020.5565

Qi, M., Yu, B., Yu, H., and Li, F. 2020. Integrated analysis of a ceRNA network reveals potential prognostic IncRNAs in gastric cancer. Cancer Medicine. 10.1002/cam4.2760

Reczek, C.R., and Chandel, N.S. 2018. ROS Promotes Cancer Cell Survival through Calcium Signaling. CANCER CELL 33:949-951. 10.1016/j.ccell.2018.05.010

Saka, M., Morita, S., Fukagawa, T., and Katai, H. 2011. Present and future status of gastric cancer surgery. JAPANESE JOURNAL OF CLINICAL ONCOLOGY 41:307-313. 10.1093/jjco/hyq240

Sang, B., Zhang, Y.Y., Guo, S.T., Kong, L.F., Cheng, Q., Liu, G.Z., Thorne, R.F., Zhang, X.D., Jin, L., and Wu, M. 2018. Dual functions for OVAAL in initiation of RAF/MEK/ERK prosurvival signals and evasion of $\mathrm{p} 27$-mediated cellular senescence. Proceedings of the National Academy of Sciences 115:E11661-E11670. 10.1073/pnas. 1805950115

Shen, J., Cao, B., Wang, Y., Ma, C., Zeng, Z., Liu, L., Li, X., Tao, D., Gong, J., and Xie, D. 2018. Hippo component YAP promotes focal adhesion and tumour aggressiveness via transcriptionally activating THBS1/FAK signalling in breast cancer. J Exp Clin Cancer Res 37:175. 10.1186/s13046-018-0850-z

Shi, X., Guo, X., Li, X., Wang, M., and Qin, R. 2018. Loss of Linc01060 induces pancreatic cancer progression through vinculin-mediated focal adhesion turnover. CANCER LETTERS 433:76-85. 10.1016/j.canlet.2018.06.015

Siegel, R.L., Miller, K.D., and Jemal, A. 2019. Cancer statistics, 2019. CA Cancer J Clin 69:7-34. $10.3322 /$ caac. 21551

Sobotka, L.A., Husain, S.G., Krishna, S.G., Hinton, A., Pavurula, R., Conwell, D.L., and Zhang, C. 2018. A risk score model of 30-day readmission in ulcerative colitis after colectomy or proctectomy. Clin Transl Gastroenterol 9:175. 10.1038/s41424-018-0039-y

Wang, J., Duncan, D., Shi, Z., and Zhang, B. 2013. WEB-based GEne SeT AnaLysis Toolkit (WebGestalt): update 2013. NUCLEIC ACIDS RESEARCH 41:W77-W83. 10.1093/nar/gkt439

Wang, M.W., Liu, J., Liu, Q., Xu, Q.H., Li, T.F., Jin, S., and Xia, T.S. 2017. LncRNA SNHG7 promotes the proliferation and inhibits apoptosis of gastric cancer cells by repressing the P15 and P16 expression. Eur Rev Med Pharmacol Sci 21:4613-4622.

Wei, G.H., and Wang, X. 2017. IncRNA MEG3 inhibit proliferation and metastasis of gastric cancer via p53 signaling pathway. Eur Rev Med Pharmacol Sci 21:3850-3856.

Yang, H.J., Choi, S., Park, S.K., Jung, Y.S., Choi, K.Y., Park, T., Kim, J.Y., and Park, D.I. 2017. Derivation and validation of a risk scoring model to predict advanced colorectal neoplasm in adults of all ages. $J$ Gastroenterol Hepatol 32:1328-1335. 10.1111/jgh.13711

Yang, M., Qin, X., Qin, G., and Zheng, X. 2019. The role of IRAK1 in breast cancer patients treated with neoadjuvant chemotherapy. Onco Targets Ther 12:2171-2180. 10.2147/OTT.S185662

Yu, C., and Zhang, Y. 2019. Development and validation of prognostic nomogram for young patients with gastric cancer. Annals of Translational Medicine 7:641. 10.21037/atm.2019.10.77

Yu, Y., Nangia-Makker, P., Farhana, L., and Majumdar, A. 2017. A novel mechanism of lncRNA and miRNA interaction: CCAT2 regulates miR-145 expression by suppressing its maturation process in colon cancer cells. Molecular Cancer 16:155. 10.1186/s12943-017-0725-5

Zeng, J., Lu, W., Liang, L., Chen, G., Lan, H., Liang, X., and Zhu, X. 2019. Prognosis of clear cell renal cell carcinoma (ccRCC) based on a six-lncRNA-based risk score: an investigation based on RNA-sequencing data. Journal of Translational Medicine 17. 10.1186/s12967-019-2032-y

Zhang, L., Wang, Y., Sun, J., Ma, H., and Guo, C. 2019. LINC00205 promotes proliferation, migration and invasion of HCC cells by targeting miR-122-5p. Pathology - Research and Practice 215:152515. 10.1016/j.prp.2019.152515

Zhang, X., Zhang, W., Jiang, Y., Liu, K., Ran, L., and Song, F. 2019. Identification of functional lncRNAs in gastric cancer by integrative analysis of GEO and TCGA data. JOURNAL OF CELLULAR BIOCHEMISTRY 120:1789817911. 10.1002/jcb.29058

Zhang, Z., Liu, Q., Wang, P., Li, J., He, T., Ouyang, Y., Huang, Y., and Wang, W. 2018. Development and internal validation of a nine-lncRNA prognostic signature for prediction of overall survival in colorectal cancer patients. PeerJ 6:e6061. 10.7717/peerj.6061

Zhao, J., Guo, C., Ma, Z., Liu, H., Yang, C., and Li, S. 2020. Identification of a novel gene expression signature associated with overall survival in patients with lung adenocarcinoma: A comprehensive analysis based on TCGA

Peer) reviewing PDF | (2020:02:45656:3:0:NEW 4 Nov 2020) 
515 and GEO databases. LUNG CANCER 149:90-96. 10.1016/j.lungcan.2020.09.014

516 Zhao, X., Sun, S., Zeng, X., and Cui, L. 2018. Expression profiles analysis identifies a novel three-mRNA signature 517 to predict overall survival in oral squamous cell carcinoma. American Journal of Cancer Research 8:450-461.

518 Zhou, H.Y., Wu, C.Q., and Bi, E.X. 2019. MiR-96-5p inhibition induces cell apoptosis in gastric adenocarcinoma. 519 World J Gastroenterol 25:6823-6834. 10.3748/wjg.v25.i47.6823

520 Zhu, M., Wang, Q., Luo, Z., Liu, K., and Zhang, Z. 2018. Development and validation of a prognostic signature for preoperative prediction of overall survival in gastric cancer patients. Volume 11:8711-8722. 10.2147/OTT.S181741 Zhu, X., Tian, X., Yu, C., Shen, C., Yan, T., Hong, J., Wang, Z., Fang, J., and Chen, H. 2016. A long non-coding RNA signature to improve prognosis prediction of gastric cancer. Molecular Cancer 15. 10.1186/s12943-016-05440

Zhuan, B., Lu, Y., Chen, Q., Zhao, X., Li, P., Yuan, Q., and Yang, Z. 2019. Overexpression of the long noncoding

RNA TRHDE - AS1 inhibits the progression of lung cancer via the miRNA - 103/KLF4 axis. JOURNAL OF

CELLULAR BIOCHEMISTRY. 10.1002/jcb.29029 


\section{Figure 1}

The expression information of five IncRNAs, overall survival and disease free survival in gastric cancer patients in the TCGA dataset.

(A) Volcano plot with blue dots indicating five IncRNAs expression levels which is significantly different between tumor and normal tissue based on the criteria of an absolute log2 fold change (FC) $>1$ and adjusted $P<0.05$. (B) Heatmap of the five-IncRNA expression profile of the 414 patients in the TCGA dataset. Among five IncRNAs, MIR100HG and TRHDE-AS1 have a similar expression in 414 patients in the TCGA dataset, otherwise the other three IncRNAs do as well. (C-D) The survival curves based on the OS and DFS of the 408 patients in TCGA dataset. 

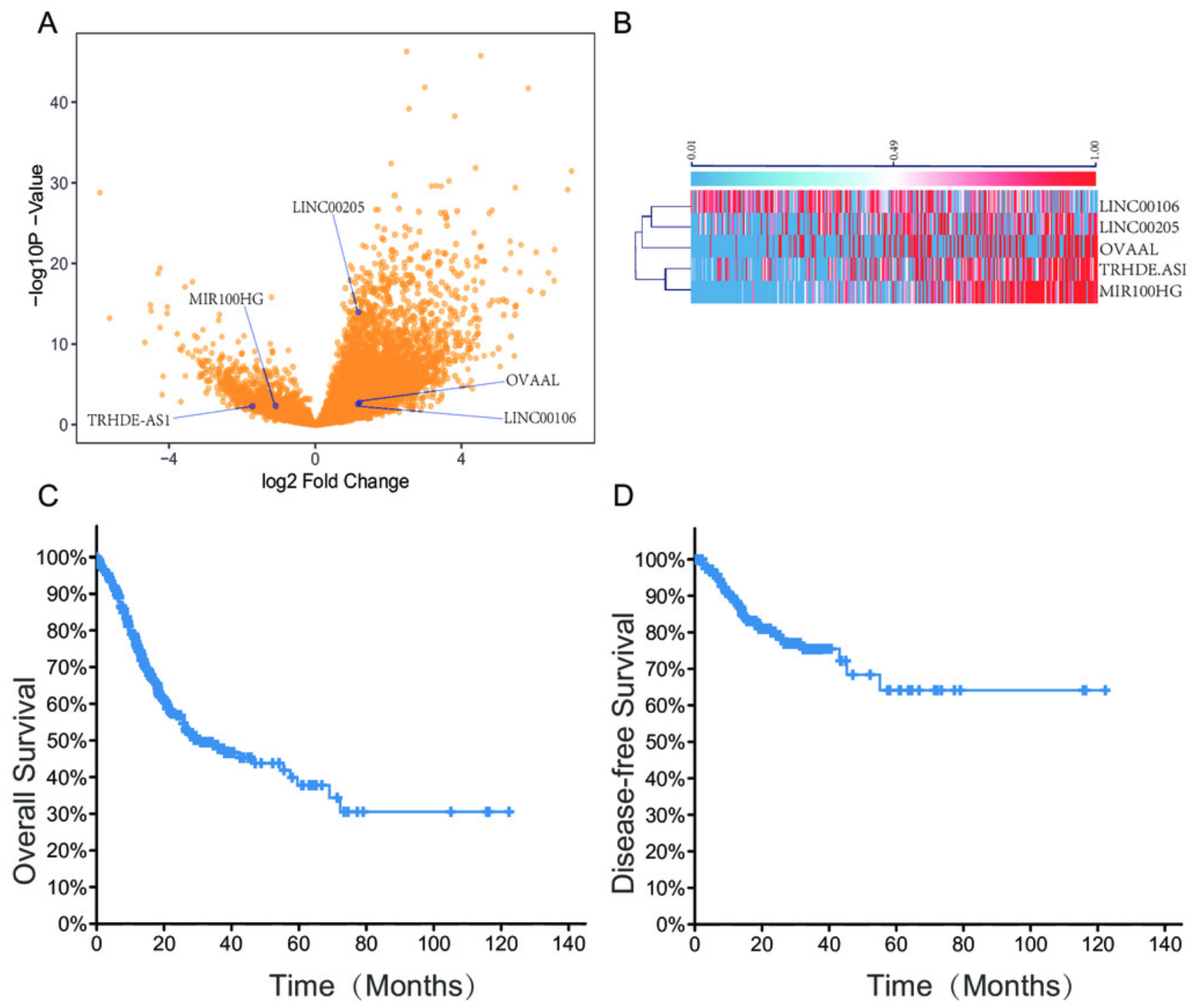


\section{Figure 2}

The prognostic value of IncRNA-based risk model in training group.

(A-B) Kaplan-Meier analysis of patients' OS and DFS in the high risk $(n=204)$ and low risk ( $n$ $=204$ ) subgroups of the training group. (C) The scatter plot of IncRNA-based risk model distribution for patient survival status. (D) The percentage of patient survival status in the high risk and low risk subgroups of the training group. (E) The IncRNA-based risk model distribution for patient recurrence. (F) The percentage of patient recurrence in the high risk and low risk subgroups of the training group. (G-H) The time-dependent ROC analysis of the risk score for prediction the 4-year cutoff OS and 2-year cutoff DFS of the training group. The area under the curve was calculated for ROC curve. ${ }^{* * *}{ }^{*}<0.001$. 

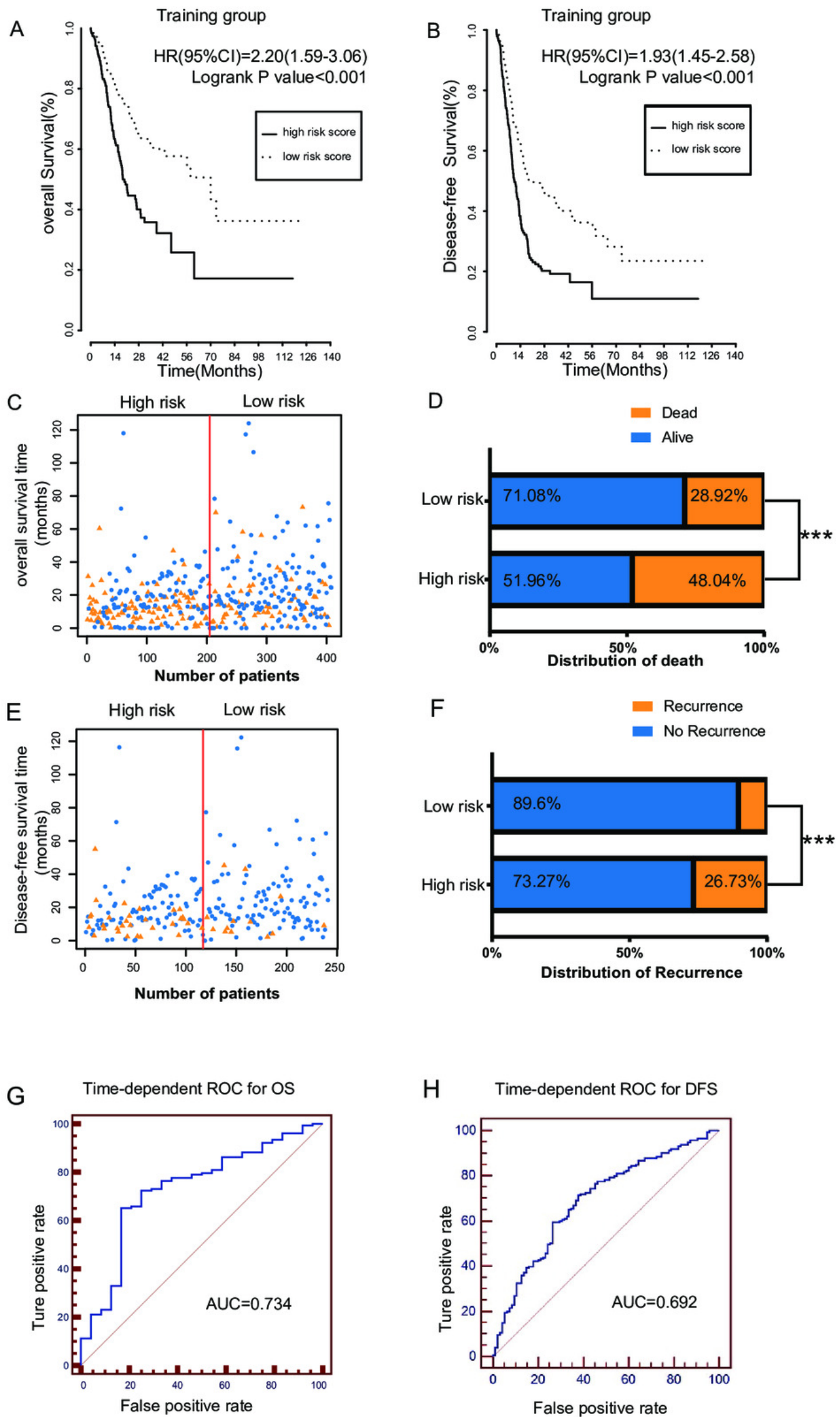


\section{Figure 3}

The prognostic value of IncRNA-based risk model in two independent GEO validation groups.

(A-B) Kaplan-Meier analysis of predicting OS of GC patients based on the high risk and low risk subgroups in two independent validation groups (GSE62254 and GSE15459). (C-D) The scatter plot of five-IncRNA-based risk score distribution for patient survival status in two independent validation groups.(E-F) The time-dependent ROC analysis of the risk score for prediction the 4-year cutoff OS of the two independent validation groups. The area under the curve was calculated for ROC curve. 
A

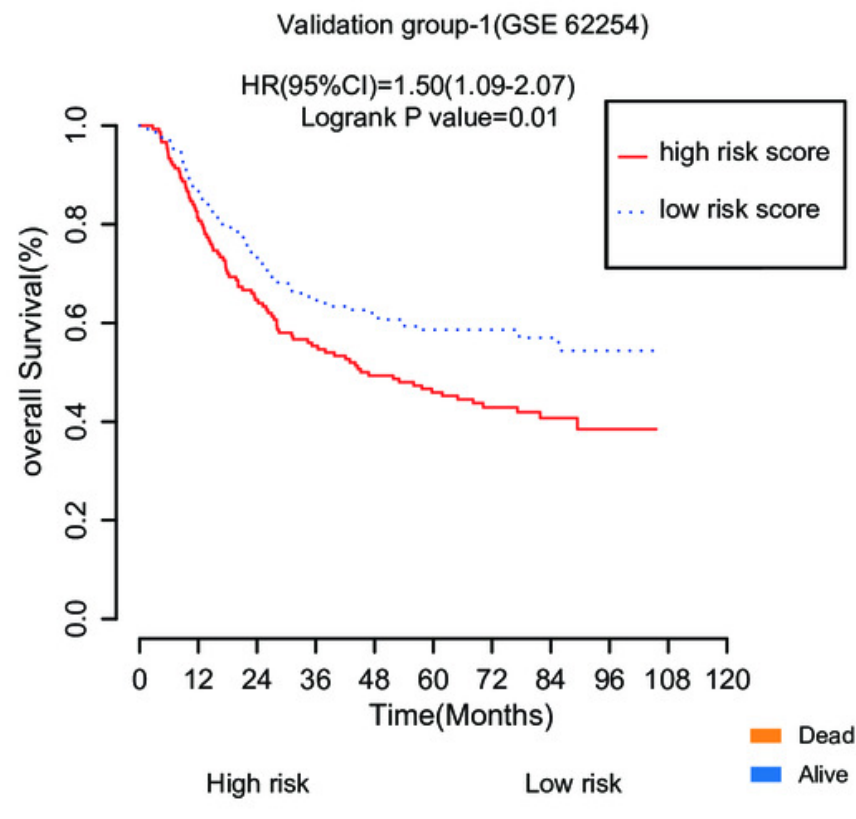

C

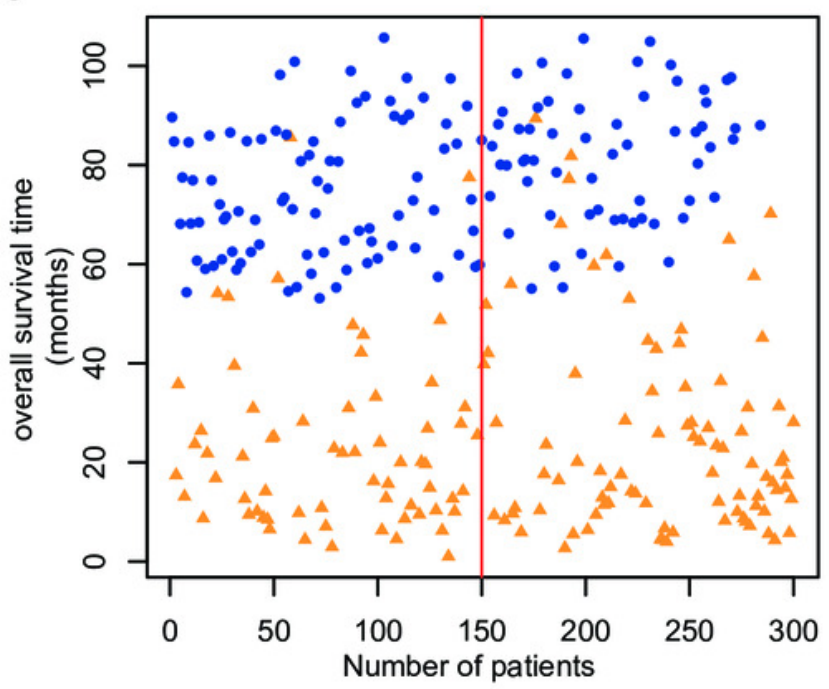

E Time-dependent ROC for group-1

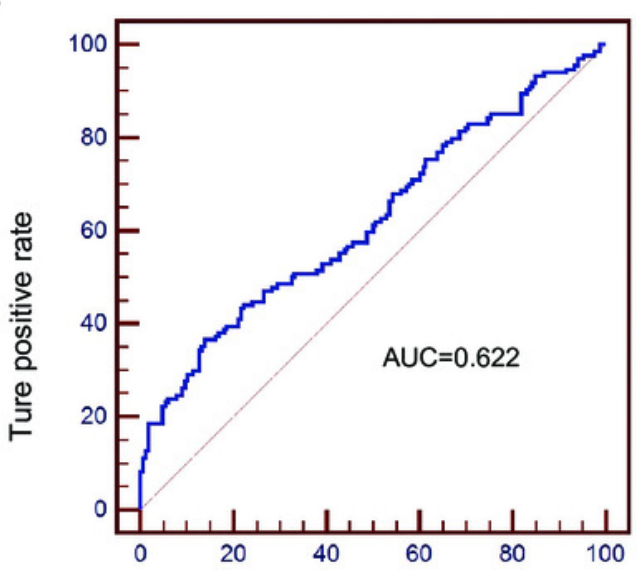

False positive rate

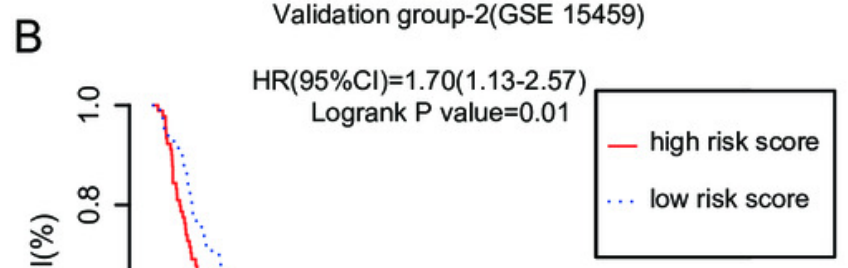

- Dead

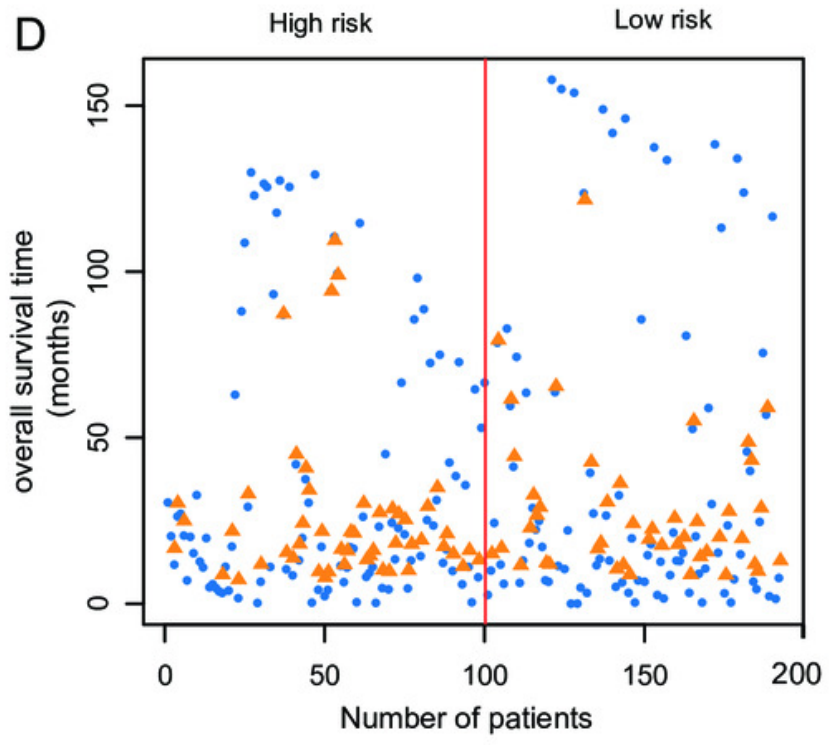

F Time-dependent ROC for group-2

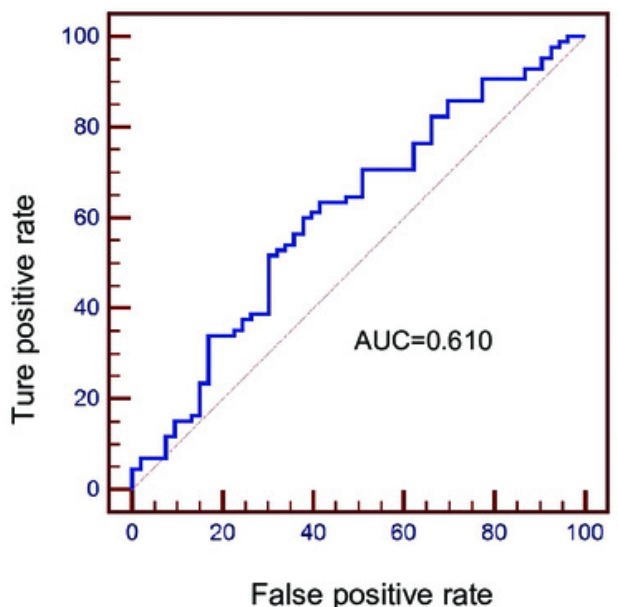

False positive rate 
Figure 4

The prognostic value of IncRNA-based risk model in subgroups according to the TNM stage.

(A-D) Kaplan-Meier analysis of the OS of GC patients with stagel, II, III and IV, respectively. 
A

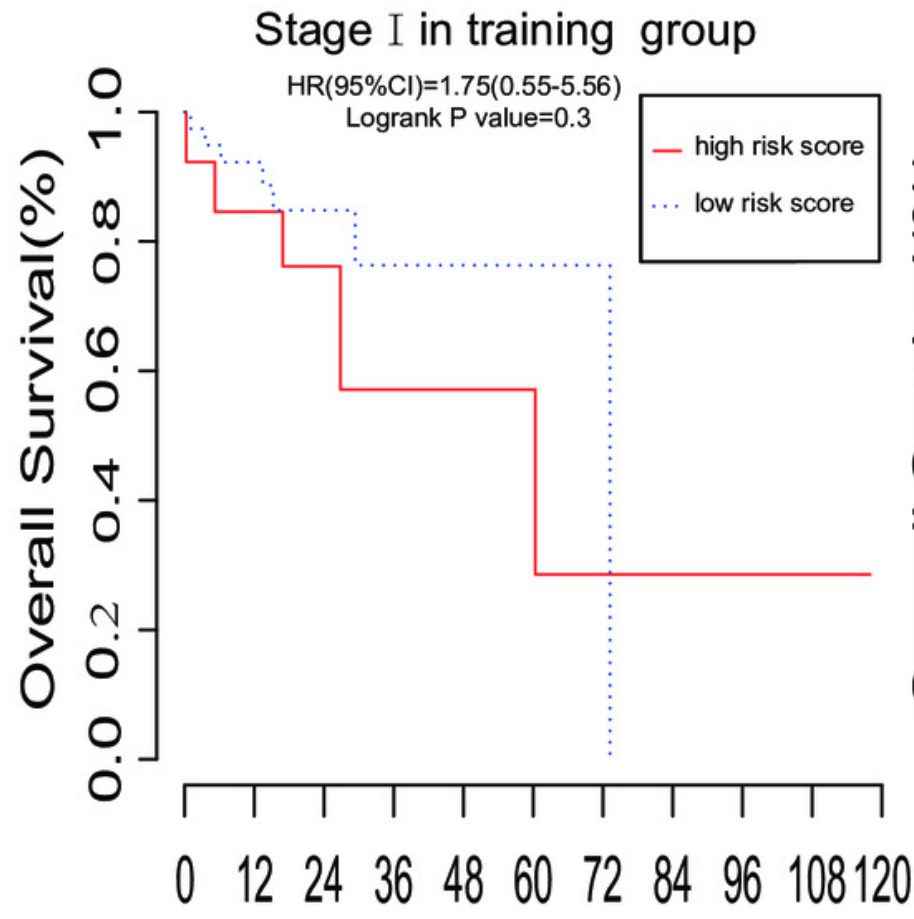

C

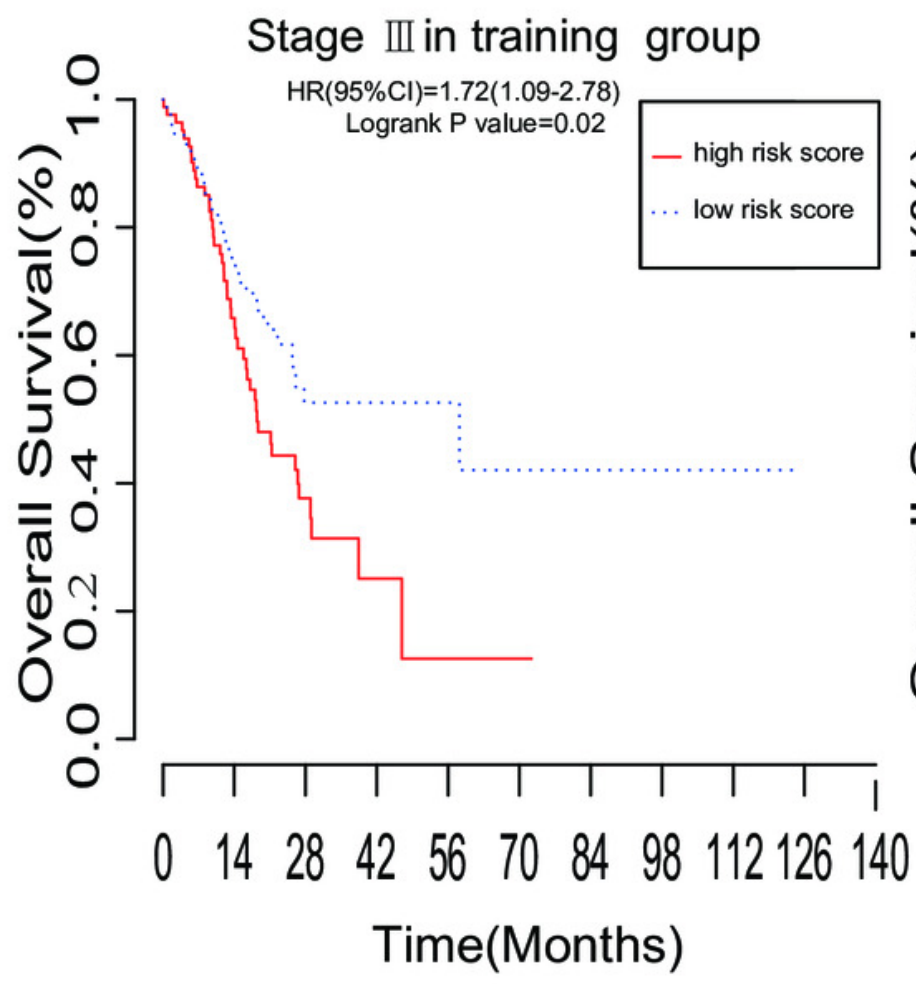

B

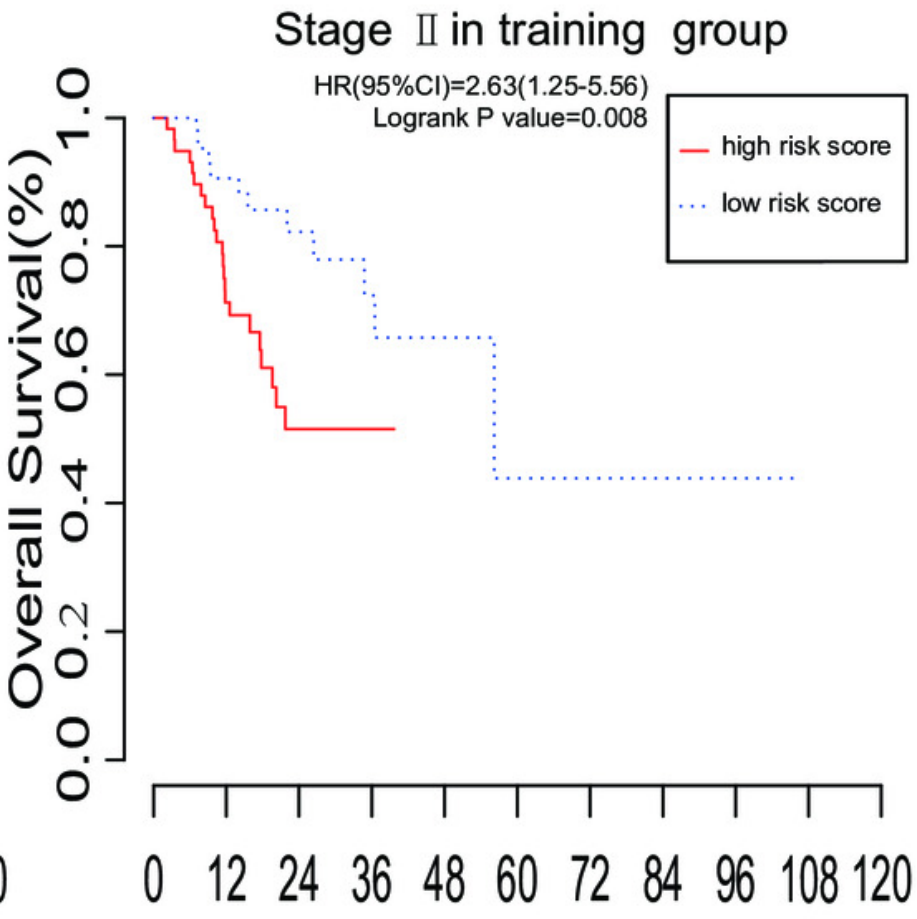

D

Time(Months)

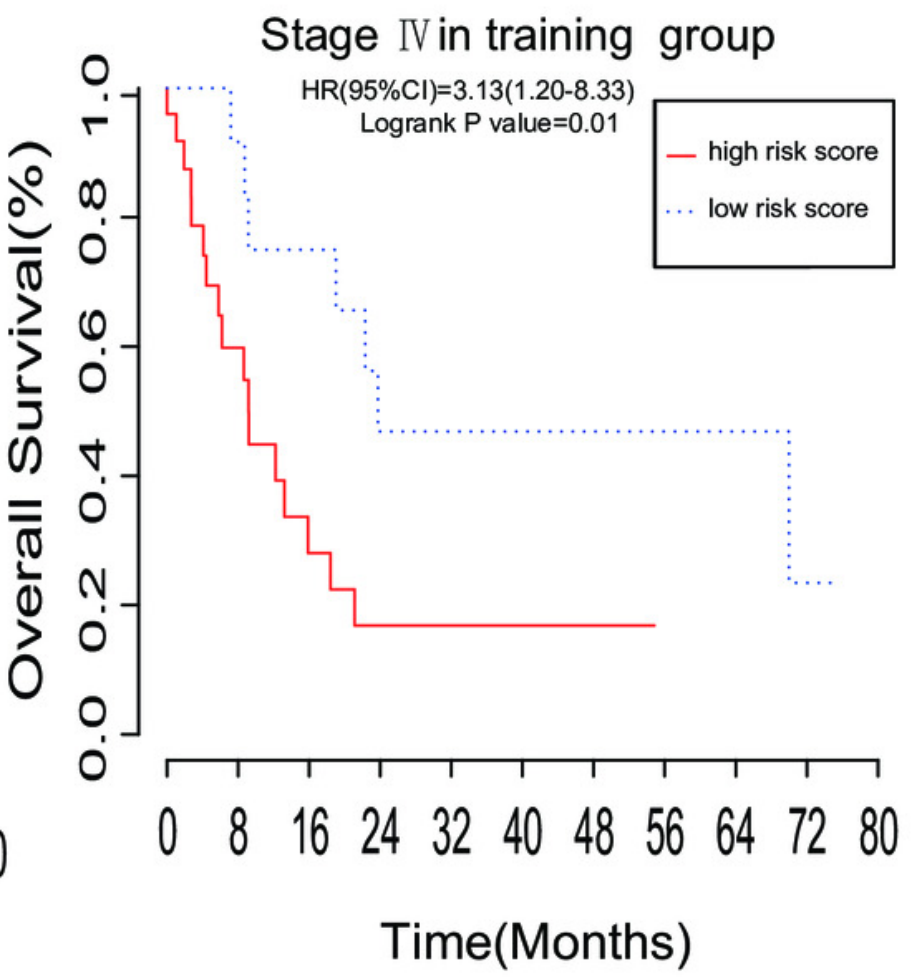


Figure 5

Forest plot to evaluate prognostic value of IncRNA-based risk model in subgroups divided by clinical factors. 


\section{Forest plot for clinic subgroup}

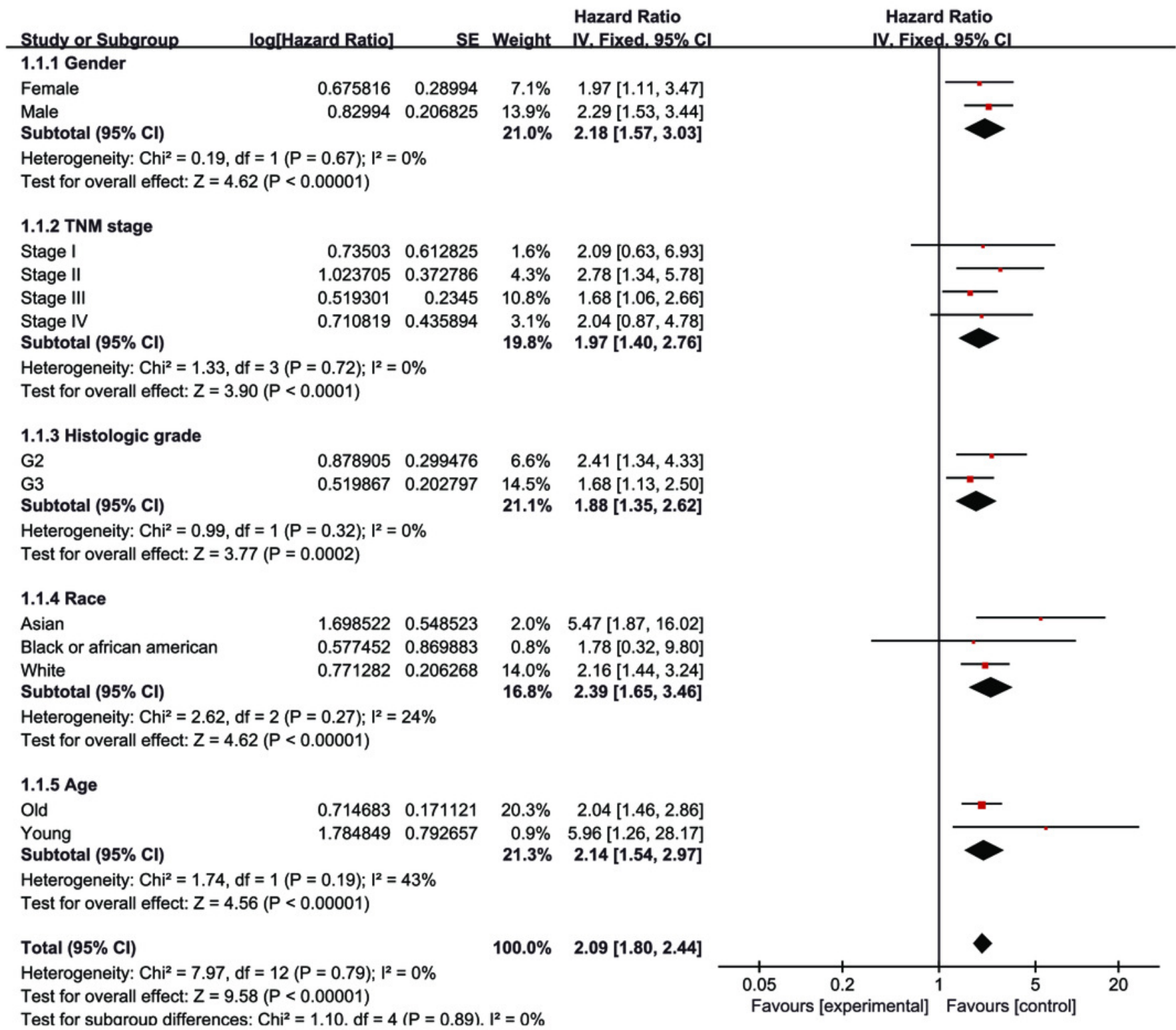




\section{Figure 6}

The prognostic value of a nomogram model combining five-IncRNA signature with the clinical factors.

(A) A nomogram model combining five-IncRNA signature with the clinical factors for predicting the 4-year OS of GC patients. (B) The nomogram calibration curve to evaluate the prediction of 4-year OS of GC patients. The C index of this model was also calculated. 
A

\section{Nomogram model}

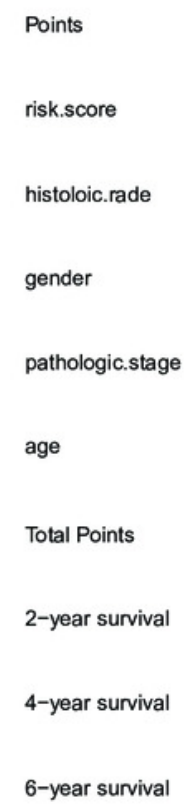

B

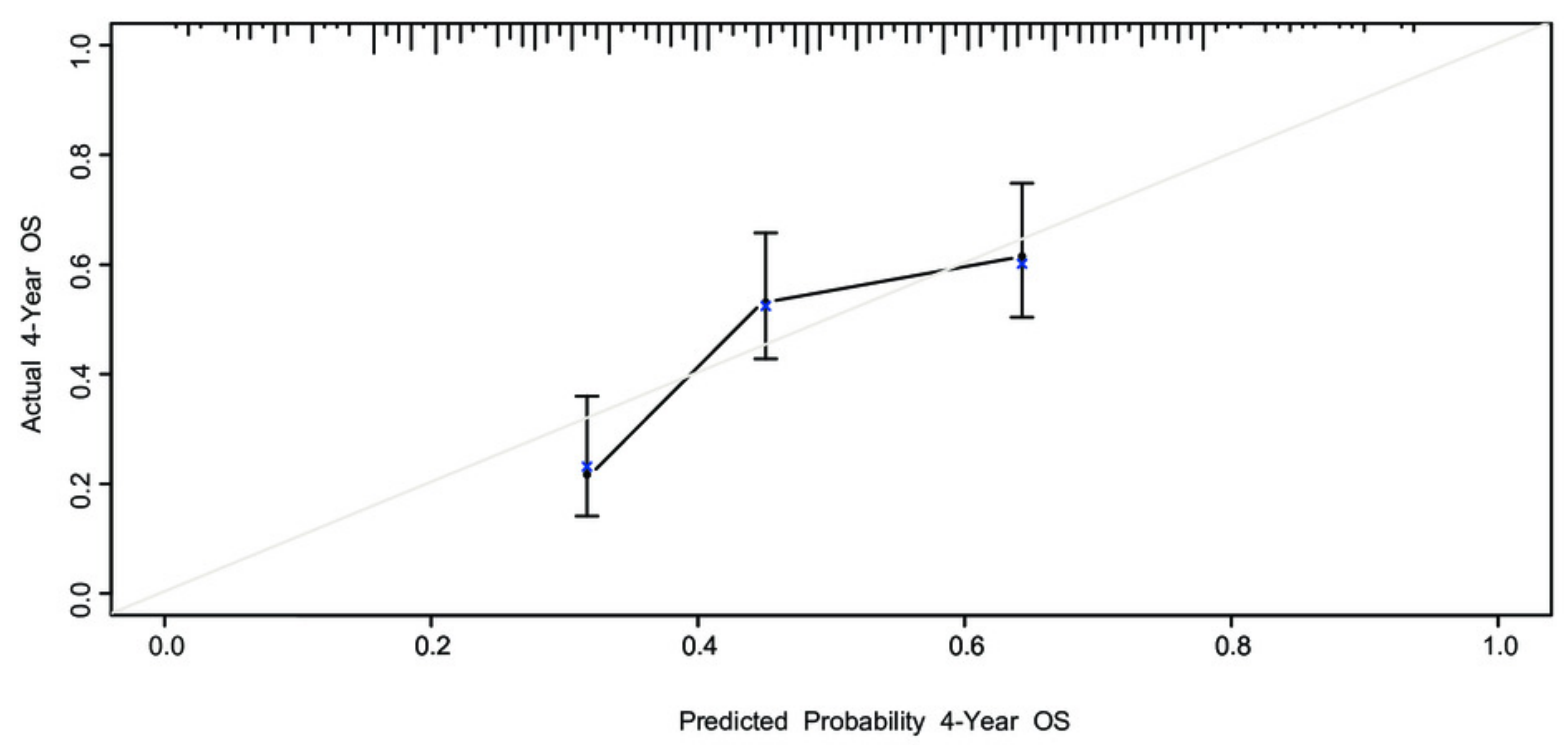


Figure 7

Potential functions of the five IncRNAs

(A) The Pearson correlation coefficient between 19605 protein-coding genes and five IncRNAs

in TCGA dataset. (B) The functional enrichment bubble map of pathways by KEGG pathway analysis. Bubble size represents the number of gene enriched in the pathway. 
A

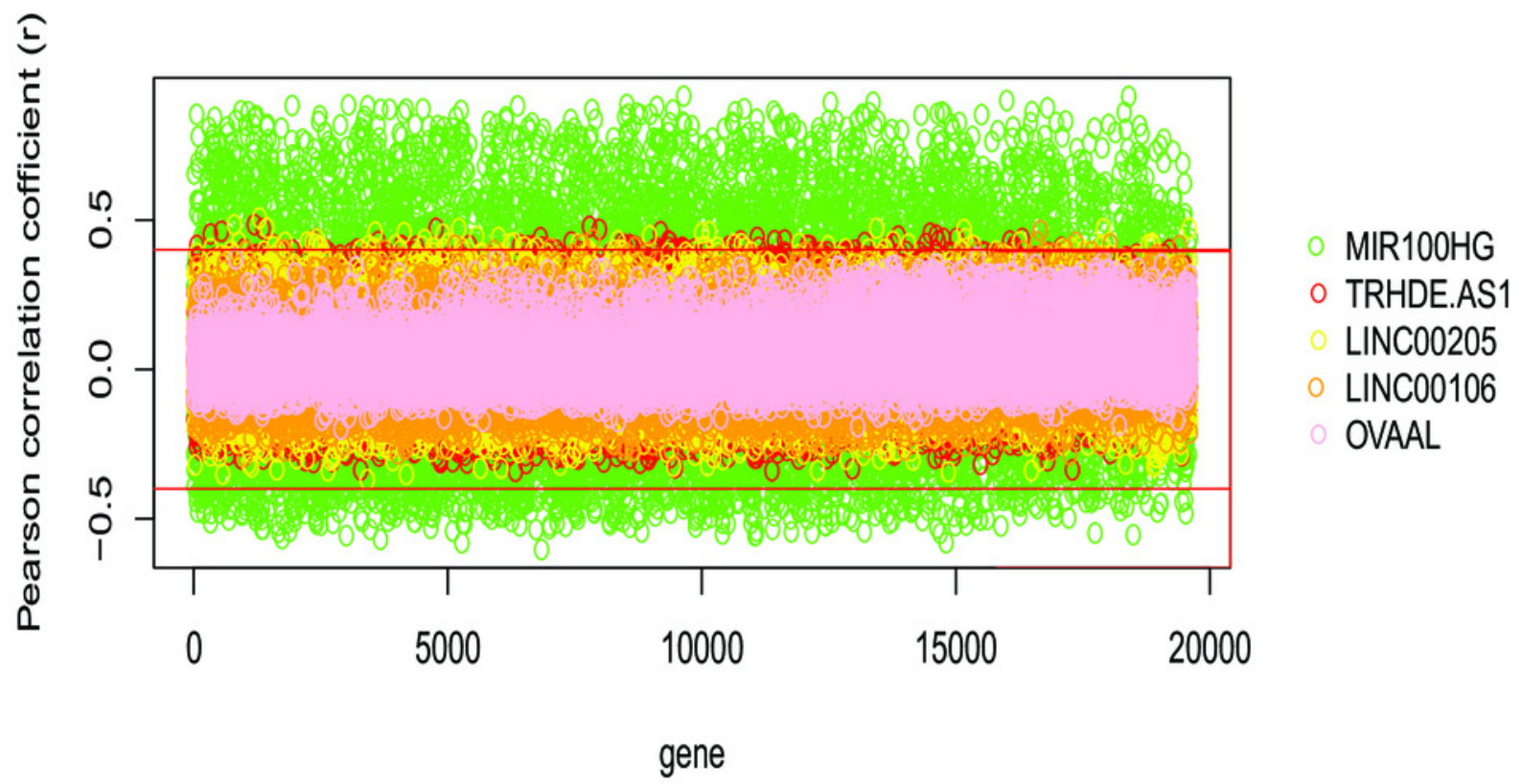

B

\section{Top10 of pathway enrichment}

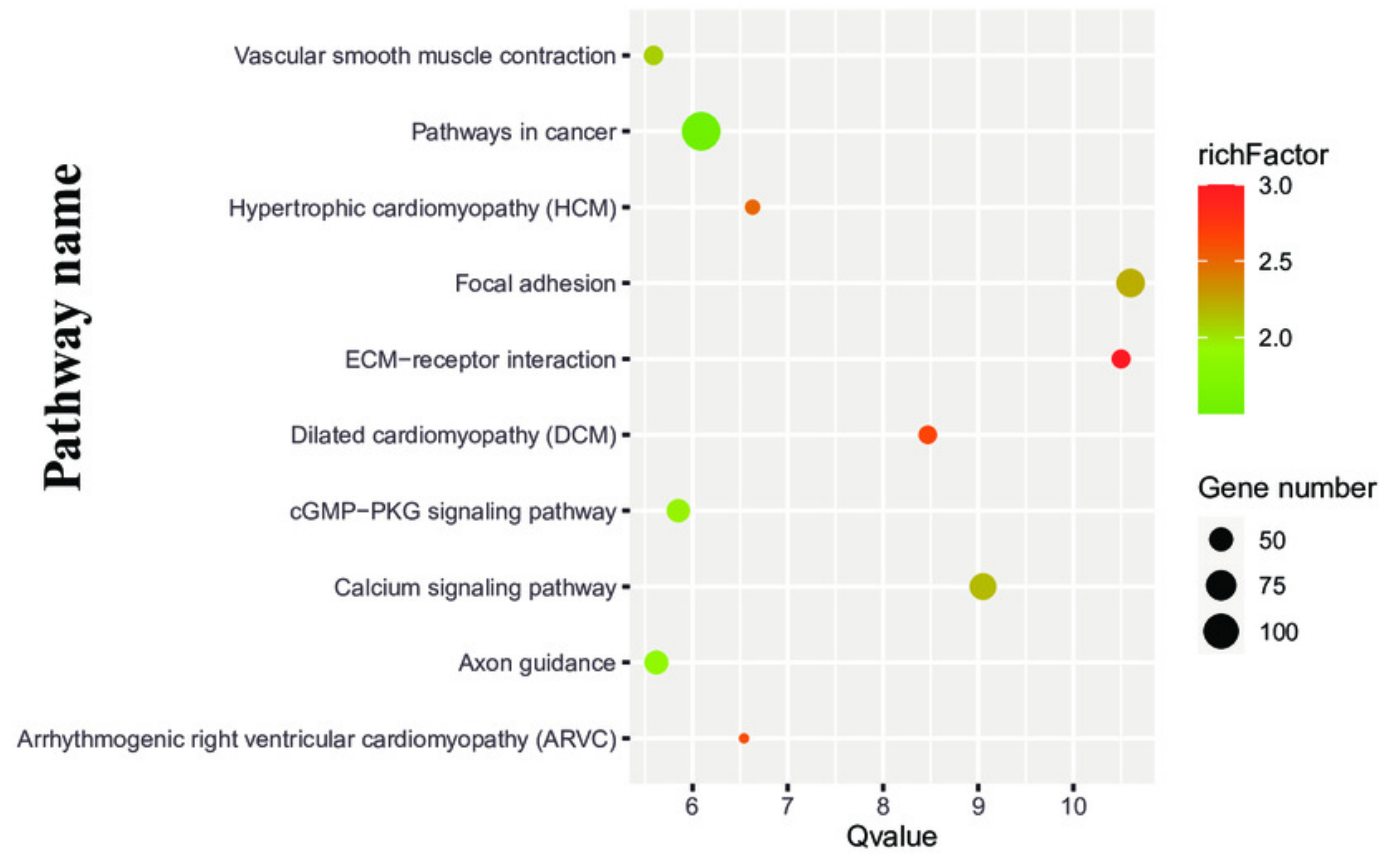




\section{Table $\mathbf{1}$ (on next page)}

Five IncRNAs significantly associated with prognosis of GC patients in the training group.

Derived from the multivariable Cox proportional hazards regression analysis in the training group. 


\begin{tabular}{ccccccc}
\hline LncRNA name & Ensemble ID & Chr. & Coordinate & Coefficient & Hazard ratio & P value \\
\hline LINC00205 & ENSG00000223768.1 & 21 & $45288052-45297354$ & 0.249092 & 1.373451497 & 0.047216345 \\
TRHDE-AS1 & ENSG00000236333.3 & 12 & $72253507-72273509$ & 0.182045 & 1.846654514 & 0.000109193 \\
OVAAL & ENSG00000236719.2 & 1 & $180558974-180566518$ & 0.271169 & 1.880897277 & 0.0000744 \\
LINC00106 & ENSG00000236871.6 & X\&Y & $1397025-1399412$ & -0.207942 & 0.624972486 & 0.003469142 \\
MIR100HG & ENSG00000255248.6 & 11 & $122028329-122422871$ & 0.502539 & 1.396343319 & 0.036829012 \\
\hline
\end{tabular}


Table 2 (on next page)

The clinical features of GC patients in training group and GSE62254. 


\begin{tabular}{|c|c|c|c|c|}
\hline \multirow[b]{2}{*}{ Variables } & \multicolumn{2}{|c|}{ Training group } & \multicolumn{2}{|c|}{$\begin{array}{c}\text { Validation group-1 } \\
\text { (GSE62254) }\end{array}$} \\
\hline & $n=408$ & $\%$ & $\mathbf{n}=\mathbf{3 0 0}$ & $\%$ \\
\hline \multicolumn{5}{|l|}{ Gender } \\
\hline Male & 263 & 64.46 & 199 & 66.33 \\
\hline Female & 145 & 35.54 & 101 & 33.67 \\
\hline \multicolumn{5}{|l|}{ Age } \\
\hline Old $(>=50$ years old $)$ & 377 & 92.40 & 262 & 87.33 \\
\hline Young $(<50$ years old $)$ & 31 & 7.60 & 38 & 12.67 \\
\hline \multicolumn{5}{|l|}{ TNM stage } \\
\hline Stage I & 55 & 13.48 & 30 & 10.00 \\
\hline Stage II & 120 & 29.41 & 96 & 32.00 \\
\hline Stage III & 167 & 40.93 & 95 & 31.67 \\
\hline Stage IV & 41 & 10.05 & 79 & 26.33 \\
\hline Not Available & 25 & 6.13 & 0 & \\
\hline \multicolumn{5}{|l|}{ T stage } \\
\hline $\mathrm{T} 1$ & 20 & 4.90 & 2 & 0.67 \\
\hline $\mathrm{T} 2$ & 87 & 21.32 & 186 & 62.00 \\
\hline $\mathrm{T} 3$ & 178 & 43.63 & 91 & 30.33 \\
\hline $\mathrm{T} 4$ & 114 & 27.94 & 21 & 7.00 \\
\hline $\mathrm{TX}$ & 9 & 2.21 & 0 & \\
\hline \multicolumn{5}{|l|}{ N stage } \\
\hline N0 & 120 & 29.41 & 38 & 12.67 \\
\hline N1 & 110 & 26.96 & 131 & 43.67 \\
\hline N2 & 77 & 18.87 & 80 & 26.67 \\
\hline N3 & 82 & 20.10 & 51 & 17.00 \\
\hline $\mathrm{NX}$ & 17 & 4.17 & 0 & \\
\hline Not Available & 2 & 0.49 & 0 & \\
\hline \multicolumn{5}{|l|}{ M stage } \\
\hline M0 & 362 & 88.73 & 273 & 91.00 \\
\hline M1 & 27 & 6.62 & 27 & 9.00 \\
\hline MX & 19 & 4.66 & 0 & \\
\hline \multicolumn{5}{|l|}{ Survival status } \\
\hline Alive & 251 & 61.52 & 148 & 49.33 \\
\hline Dead & 157 & 38.48 & 152 & 50.67 \\
\hline
\end{tabular}


Table 3 (on next page)

The schematic workflow of the present study. 
(1) LncRNA expression profiles from TCGA database (450 GC samples;14147 IncRNAs)

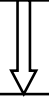

(2) Bayesian analysis and univariate cox proportional hazard regression analysis identified 278 IncRNAs involved

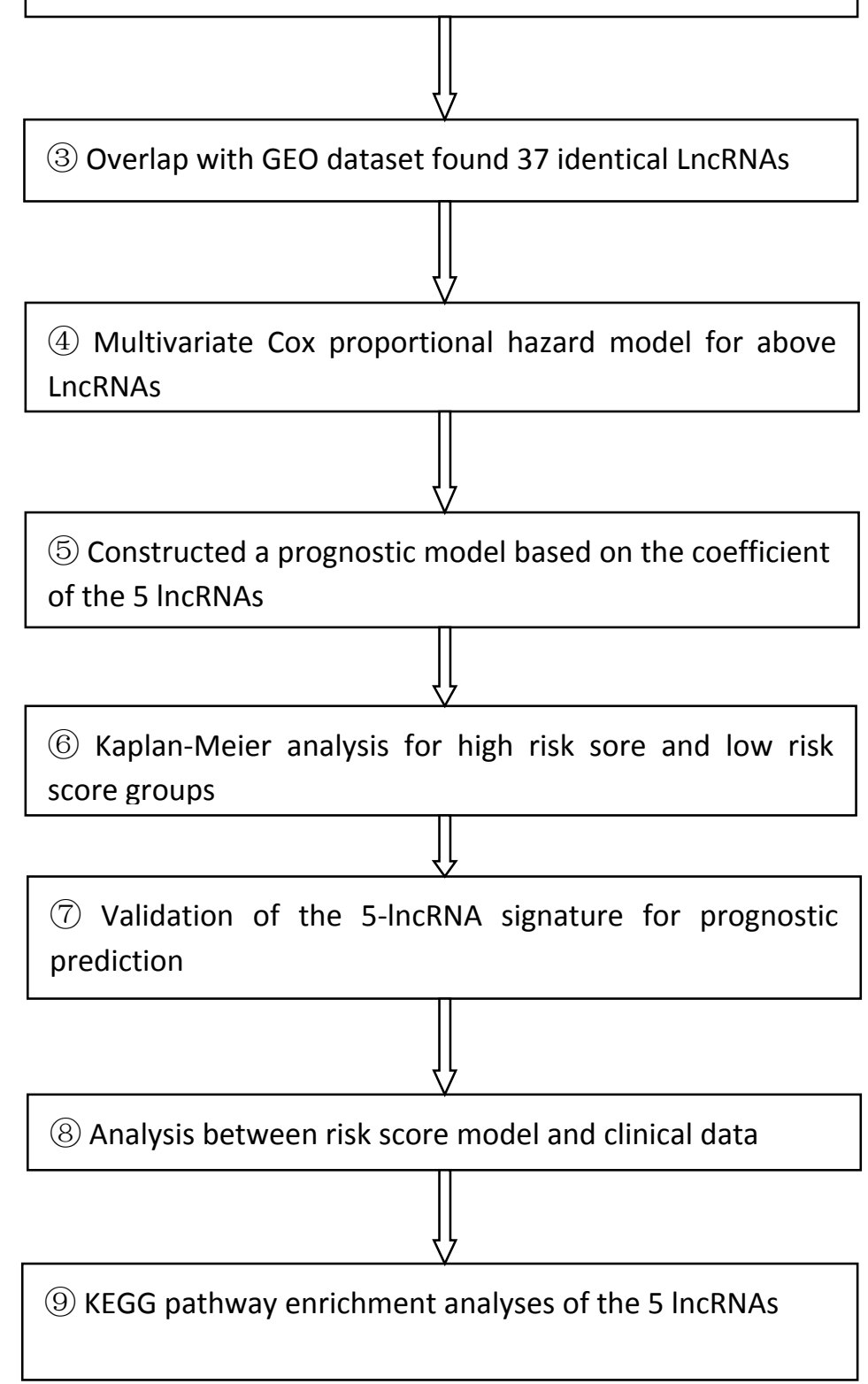




\section{Table 4 (on next page)}

The association between five-IncRNA signature and OS of GC patients in training group.

Abbreviations: $\mathrm{HR}$, Hazard ratio; $95 \% \mathrm{Cl}, 95 \%$ confidence interval. 


\begin{tabular}{|c|c|c|c|}
\hline & $\begin{array}{c}\text { Number (High } \\
\text { Risk score/Low } \\
\text { Risk score) }\end{array}$ & $\mathrm{HR} \quad(95 \% \mathrm{CI})$ & $\mathrm{P}$ value \\
\hline Total & $204 / 204$ & $2.09(1.80,2.44)$ & 0.000001 \\
\hline \multicolumn{4}{|l|}{ Gender } \\
\hline Male & $129 / 134$ & $2.29(1.53,3.44)$ & 0.00002 \\
\hline Female & $75 / 70$ & $1.97(1.11,3.47)$ & 0.01 \\
\hline \multicolumn{4}{|l|}{ Histologic grade } \\
\hline $\mathrm{G} 2$ & $47 / 97$ & $2.41(1.34,4.33)$ & 0.0006 \\
\hline G3 & $146 / 97$ & $1.68(1.13,2.50)$ & 0.02 \\
\hline \multicolumn{4}{|l|}{ Race } \\
\hline Asian & $44 / 41$ & $\begin{array}{c}5.47 \quad(1.87, \\
16.02)\end{array}$ & 0.001 \\
\hline $\begin{array}{l}\text { Black or african } \\
\text { american }\end{array}$ & $4 / 8$ & $1.78(0.32,9.80)$ & 0.6 \\
\hline White & $138 / 120$ & $2.16(1.44,3.24)$ & 0.0003 \\
\hline \multicolumn{4}{|l|}{ Age } \\
\hline 01d $\quad(>=50$ years old $)$ & $186 / 191$ & $2.04(1.46,2.86)$ & 0.00001 \\
\hline Young ( $<50$ years old $)$ & $18 / 13$ & $\begin{array}{c}5.96(1.26 \\
28.17)\end{array}$ & 0.008 \\
\hline \multicolumn{4}{|l|}{ TNM stage } \\
\hline Stage I & $14 / 41$ & $2.09(0.63,6.93)$ & 0.3 \\
\hline Stage II & $62 / 58$ & $2.78(1.34,5.78)$ & 0.008 \\
\hline Stage III & $87 / 77$ & $1.68(1.06,2.66)$ & 0.02 \\
\hline Stage IV & $25 / 16$ & $2.04(0.87,4.78)$ & 0.01 \\
\hline
\end{tabular}

1 